\title{
Accounting for Dispersion Effects in DFT Framework of Electrocatalysis: A Case Study of Solvent Mediated Oxygen Reduction Reaction
}

\author{
Akhil S. Nair ${ }^{\dagger}$ Biswarup Pathak ${ }^{\dagger^{*}}$ \\ 'Department of Chemistry, Indian Institute of Technology Indore, Simrol, Indore 453552, India
}

Email: biswarup@iiti.ac.in

\begin{abstract}
Density functional theory (DFT) is a pivotal tool in the field of computational electrocatalysis. Dispersion effects, which are not incorporated in the regular DFT framework, play a significant role in improving the accuracy of DFT-based catalytic simulations. We perform a calibration study for addressing the effect of different dispersion corrected DFT methods in determining the electrocatalytic properties by conducting a case study of oxygen reduction reaction (ORR). The distinct trends of these methods towards determining the structural, energetic, and electronic properties of catalysis are scrutinized. By systematically incorporating an upgraded solvation model, the importance of the inclusion of dispersion effects for the accurate prediction of chemical and physical properties governing the catalytic activity is illustrated. The combined thermodynamic and kinetic analysis predicts a uniform ORR activity trend, with semi-empirical dispersion corrected DFT methods emerging as optimal choices with comparable or higher accuracy than advanced van der Waal's methods.
\end{abstract}

Keywords: Oxygen reduction reaction, dispersion, solvation, kinetics, $\mathrm{Pt}(111)$ surface

\section{Introduction}

Electrocatalysis has been appraised as an area of tremendous importance owing to its supreme role in empowering the development of renewable energy-related materials to meet the proliferating energy demand. ${ }^{1,2}$ Very recent decades have witnessed profound advancement in this area by introducing fuel cells, batteries, hydrogen storage materials, and photocatalysts to resolve the burgeoning energy crisis by successfully utilizing the results obtained from extensive studies on electrocatalytic reactions. ${ }^{3-5}$ Oxygen reduction reaction (ORR) is one of the well-known and widely studied electrocatalytic reactions occurring in the cathode of proton exchange membrane (PEM) fuel cells. It is the sluggish nature of ORR which renders challenges in attaining the expected efficiency of the fuel cell in spite of its desirable properties like high thermodynamic 
efficiency, energy density and zero emission. ${ }^{6-8} \mathrm{Pt}$ based catalysts retain the dominancy in fuel cell catalysis regime irrespective of development of several non-Pt catalysts because of the vulnerable nature of later in fuel cell working conditions. ${ }^{9}$ Therefore scrutinizing ORR activity of Pt based catalysts is a core objective of fuel cell research. Ample attempts to unravel the complex mechanism and improve the energetics of ORR have been carried out so forth, among which computational studies, especially within the framework of density functional theory (DFT), have contributed to a significant extent. For example, the first theoretical explanation for the origin of ORR overpotential generation was given by Norskov et al. ${ }^{10}$ grounded on the binding energy descriptor-based approach along with a screening of activity of various catalysts. An impactful experimental validation of this study was done by Stamenkovic et al. ${ }^{11}$ by synthesizing alloy-based catalysts with lesser binding energy for ORR intermediates in $0.1 \mathrm{M} \mathrm{HClO}_{4}$ at room temperature. The study reported that the single crystal surface of $\mathrm{Pt}_{3} \mathrm{Ni}(111)$ showing 10 times higher ORR activity than $\mathrm{Pt}(111)$ surface and 90 times higher than $\mathrm{Pt} / \mathrm{C}$ catalysts. This has set off numerous experiments for developing efficient catalysts by alloying Pt with carefully selected other metals. Similarly, Kattel and Wang, in their theoretical study, ${ }^{12}$ observed a lowering of the binding energy of intermediates and activation of elementary steps of ORR on a strained Pt (111) surface compared to the unstrained surface. Similar theoretical studies have paved the way for developing core-shell, heteroatom doped, and nanocluster-based catalysts that have been found to enhance the ORR energetics stemming from possession of strained surfaces. ${ }^{13-15}$ Moreover, considerable efforts from theoretical studies have been succeeded in underpinning the complex mechanism and analyzing the kinetics along different pathways of ORR. ${ }^{16,17}$ Thus, a significant extent of experimental validations and legitimate explanations of catalytic activity suggest the requisite importance of theoretical studies exploring the new dimensions of fuel cell catalysis.

The state-of-the-art DFT studies have successfully predicted many catalytically relevant features such as adsorption energetics, scaling relationships, thermodynamics, and kinetics. One of the important drawbacks with these DFT methods is the inadequacy of exchange-correlation (XC) functional to account for the non-local, long-range dispersion interactions, which are vital in accurately determining the catalytic interactions and reaction mechanisms. ${ }^{18}$ Robust wave function-based approaches like coupled-cluster theory and quantum Monte Carlo (QMC) simulations can facilitate an accurate determination of correlation energy. However, the high computational cost associated with such methods limits their applications to smaller systems. In 
this respect, different affordable models and methods have been proposed and widely used for treating dispersion interactions in surface catalysis studies. Grimmie et al. proposed the DFT-D methods ${ }^{19,20,}$ which involve the addition of semi-empirical correction term using interatomic potentials for a better description of long-range interactions. Later, non-local van der Waals density functional (vdw-DF) methods were introduced by Dion et al. ${ }^{21}$, which calculates the non-local correlation energy without any empirical considerations. These methods have been reported to work well to describe layered systems such as graphite and metal-organic frameworks. ${ }^{22}$ Furthermore, the recent methodological advancements have given rise to methods like many-body dispersion energy method (MBD@ rsSCS) based on random-phase approximation by Tkatchenko et al. ${ }^{23}$ and dispersion-corrected atom-centered pseudopotential method by von Lilenfield et al. ${ }^{24}$, which take account of long-range electron correlation with higher accuracy. These dispersion corrected DFT methods have been scrutinized for their accuracy in the prediction of properties of catalytic interest. First and foremost, these methods significantly differ in the prediction of structure-related properties of catalyst surfaces. In particular, despite the improvement in a better description of adsorption scenario, vdw-DF functionals have been known for wrongly predicting the lattice constants of metals owing to their repulsive behavior at short interatomic distances. ${ }^{25}$ Yuan et al. reported a higher accuracy in adsorption energies of $\mathrm{HCOOH} / \mathrm{HCOO}$ species on $\mathrm{Pt}(111)$ surface with reference to experiment by using both semiempirical dispersion correction methods and optimized van der Waals functional based methods. ${ }^{26}$ Though van der Waals interactions are more dominant for physisorption of organic molecules such as benzene, naphthalene, variations in dispersion correction methods have been significantly affecting the interactions of smaller molecules. For instance, a high agreement in $\mathrm{CO}_{2}$ adsorption energy on $\mathrm{Cu}$ (111) surface is obtained by using PBE-D2 and optB86b methods within the experimental range. ${ }^{27}$ Apart from the changes in the interaction of intermediates with catalyst surface, the catalytic reaction mechanism has also been affected by the inclusion of dispersion interactions. Pham et al. have studied the methanol dissociation kinetics on Pt(111) surface by using PBE and optB86bvdW methods. ${ }^{28}$ It has been found that without any dispersion interactions, methanol tends to desorb from the surface, whereas the inclusion of dispersion effects predicts a low activation barrier of $0.18 \mathrm{eV}$ for the $\mathrm{C}-\mathrm{H}$ dissociation pathway in agreement with experimental observation. ${ }^{28}$ Hence, a scrutinization of dispersion corrected DFT methods subjected to the information about 
catalyst system, reactive intermediates and reaction pathways is requisite for accurate prediction of catalytically relevant parameters within the DFT framework.

Though analyzing the effect of dispersion interactions in catalysis has been an objective of many past theoretical studies, the studies so far have been majorly limited to reactions predominantly involving weak physisorption. As a result, the effect of different methods has not been much explored for industrially important electrocatalytic reactions like ORR, OER, HER, and so on, which mainly involve the chemisorption of intermediates. However, since these reactions are characterized by the electrode-electrolyte media where solvents continuously interact with the intermediates, the overall energetics and activity of such reactions can significantly contribute to dispersion interactions. ${ }^{29,30}$ Water, the essential solvent mediating ORR, has been shown to have high sensitivity towards the dispersion correction method in determining the interaction with surfaces. ${ }^{31-33}$ Michaelides and co-workers have reported that non-local vdw-DF functionals improve the water-binding on metal surfaces considerably compared to a non-van der Waals GGA framework. ${ }^{31}$ Since the structure and the interaction of water molecules on the surface can be decisive in determining the energetics of reaction occurring on the surface, such reactions can also be expected to be associated with a sensitivity towards the method of dispersion correction. Furthermore, the studies conducted in this perspective were limited to focus on structural and adsorption-related properties without exploring the detailed mechanism of ORR. ${ }^{34,35}$ Moreover, several studies have also attempted to study ORR without considering the effect of dispersion interactions. ${ }^{36-38}$ Therefore, a study scrutinizing the role of dispersion interactions and the compatibility of different available DFT methods in predicting solvent-mediated detailed mechanisms of ORR activity deserves immense attention.

Inspired by the aforementioned ideas, our current study focuses on the role of dispersion interactions in determining the ORR activity of $\mathrm{Pt}(111)$ surface by adapting different dispersion corrected DFT methods. The choice of the catalyst system is based on its versatility of $\operatorname{Pt}(111)$ as a fuel cell catalyst. ${ }^{39,40}$ By implementing a hybrid solvation model, we unravel how the dispersion interactions driving the electrode-electrolyte interface are described with different methods. A systematic analysis of the structural features of the catalyst interface is carried out. A comparison of gas phase and solvent phase scenarios is carried out for understanding the effect of dispersion interactions. Thermodynamic and kinetic features governing the ORR activity are studied, and a 
screening of different methods is carried out based on their coherence with experimental results. All the calculated results using various methods are compared and discussed with the available theoretical/experimental data. The results propose that ORR activity on $\operatorname{Pt}(111)$ surface can be significantly controlled by the choice of the dispersion correction method. A uniform activity trend is predicted from both thermodynamic and kinetic analysis of ORR reaction mechanism. Semiempirical dispersion correction methods are found to predict ORR relevant properties with comparable or better accuracy than vdw-DF methods, which validates the reliability of their wide usage in computational catalysis. The obtained trends in ORR properties can be expected to be relevant for other catalyst systems.

\section{Computational Methodology}

\subsection{Theoretical Framework of Dispersion Corrected DFT Methods}

The selected DFT methods in this work are PBE ${ }^{41}, \mathrm{PBE}^{\mathrm{D}} 2^{19}, \mathrm{PBE}^{\mathrm{D}} 3^{20}, \mathrm{RPBE}^{42}, \mathrm{RPBE}-\mathrm{D} 2^{19}$, RPBE-D3 ${ }^{20}$, vdW-DF ${ }^{21}$, vdW-DF2 ${ }^{43}$, optPBE, optB88 and optB86b ${ }^{44,45}$ which are implemented in Vienna Ab-initio simulation package (VASP). The functionals PBE and RPBE are considered as dispersion-excluded reference functionals on account of their vast application in computational heterogeneous catalysis. So far, a large number of theoretical studies with generalized gradient approximation (GGA) has been based on Grimmie's semi-empirical DFT-D dispersion correction methods. ${ }^{19,20}$ DFT-D methods hinge upon incorporating a semi-empirical correction to the energy obtained from DFT calculation to account for the dispersion interactions. In the DFT-D2 method, the dispersion included total energy is given by

$$
\mathrm{E}_{\mathrm{DFT}-\mathrm{D} 2}=\mathrm{E}_{\mathrm{KS}-\mathrm{DFT}}+\mathrm{E}_{\mathrm{D} 2}^{\mathrm{disp}}
$$

where, $\mathrm{E}_{\mathrm{KS}-\mathrm{DFT}}$ is the energy obtained by solving Kohn-Sham equations at a chosen exchangecorrelation functional $(\mathrm{XC})$ and $\mathrm{E}_{\mathrm{disp}}$ is a semi-empirical correction given by,

$$
\mathrm{E}_{\mathrm{D} 2}^{\mathrm{disp}}=-\mathrm{s}_{6} \sum_{\mathrm{i}=1}^{\mathrm{N}_{\mathrm{at}}-1} \sum_{\mathrm{j}=\mathrm{i}+1}^{\mathrm{N}_{\mathrm{at}}} \frac{\mathrm{C}_{6}^{\mathrm{ij}}}{\mathrm{R}_{\mathrm{ij}}^{6}} \mathrm{f}_{\mathrm{dmp}}\left(\mathrm{R}_{\mathrm{ij}}\right)
$$


where, $\mathrm{s}_{6}$ is a scaling factor depending on the $\mathrm{XC}$ functional, $\mathrm{N}_{\mathrm{at}}$ is the number of atoms, $\mathrm{C}_{6}^{\mathrm{ij}}$ is the coefficient of dispersion for a pair of atoms $\mathrm{i}, \mathrm{j}$ and $\mathrm{R}_{\mathrm{ij}}$ is the distance between atoms. $f_{\mathrm{dmp}}\left(\mathrm{R}_{\mathrm{ij}}\right)$ stands for a damping function to eliminate singularities at short values of $R_{i j}$.

The DFT-D3 method involves a modification in the DFT-D2 method by incorporating three-body effects and can be represented as,

$$
\begin{aligned}
& \mathrm{E}_{\mathrm{DFT}-\mathrm{D} 3}=\mathrm{E}_{\mathrm{KS}-\mathrm{DFT}}+\mathrm{E}_{\mathrm{D} 3}^{\mathrm{disp}} \\
& \mathrm{E}_{\mathrm{D} 3}^{\mathrm{disp}}=\mathrm{E}_{\mathrm{D} 2}^{\mathrm{disp}}+\mathrm{E}^{3}
\end{aligned}
$$

with the three-body energy term is given by,

$$
E^{3}=\sum_{A B C} f_{d,(3)}\left(r_{A B C}\right) E^{A B C}
$$

where the sum extends over all triple pairs of atoms $\mathrm{ABC}$ in the system. The damping function is described by geometric average radii $\mathrm{r}_{\mathrm{ABC}}$ of the system. The computational cost does not rise to higher orders even after the inclusion of D3 correction as it is majorly contributed by the time required to solve the Kohn-Sham equations. Though D3 improves the description of weak interactions for non-covalently bound complexes and metallic systems compared to D2, both of these methods have been prevailingly used for ORR studies. ${ }^{46,47}$

On the contrary to the semi-empirical methods, van der Waals density functionals (vdw-DF) include a non-local correction term incorporated to the exchange correlational functional, which can be represented as,

$$
\mathrm{E}_{\mathrm{xc}}[\mathrm{n}]=\mathrm{E}_{\mathrm{x}}^{\mathrm{GGA}}+\mathrm{E}_{\mathrm{c}}^{\mathrm{LDA}}+\mathrm{E}_{\mathrm{c}}^{\mathrm{nl}}
$$

where the first two terms on the right-hand side stand for GGA exchange energy and LDA local correlation energy, and the last term represents the non-local correlation energy, which accounts for the dispersion effects. This non-local correlation energy term can be represented as;

$$
\mathrm{E}_{\mathrm{c}}^{\mathrm{nl}}=\frac{1}{2} \int \mathrm{dr} \int \mathrm{dr} \mathrm{r}^{\prime} \mathrm{n}(\mathrm{r}) \Phi\left(\mathrm{r}, \mathrm{r}^{\prime}\right) \mathrm{n}\left(\mathrm{r}^{\prime}\right)
$$


which is calculated over electron densities at different locations interacting via a response function. Within this framework, different vdW-DF methods have been proposed by varying the exchange functionals. The original vdw-DF and vdw-DF2 methods employ revPBE and rPW86 exchange functionals, respectively, and have successfully provided a better description of interactions in dimers. ${ }^{48}$ In this work, these methods are named revPBE and rPW86, respectively, to avoid confusion between the broader vdW-DF term. The optPBE ${ }^{44}$, optB $88^{49}$ and optB $86 b^{50}$ methods are vdw-DF methods with optimized PBE, Becke88, and Becke86 functionals respectively for determining the exchange energy contribution. These methods have been reported to behave differently in terms of predicting structural parameters, binding curves and adsorption characteristics. Since DFT-D2 and D3 methods involve only improved van der Waals interaction prediction, whereas vdW-DF methods differ in terms of the exchange functionals, the terms method and functional are used interchangeably in this study.

\subsection{Computational Details of DFT Calculations}

The DFT calculations are carried out utilizing the projector augmented wave (PAW) method under periodic boundary conditions. ${ }^{51,52}$ Plane waves with an energy cutoff of $470 \mathrm{eV}$ are employed for expanding wavefunctions. A conjugate gradient algorithm is used for performing ionic relaxations with a tolerance of $10^{-4} \mathrm{eV}$ for minimum energy and $0.02 \mathrm{eV} \AA^{-1}$ for Hellmann-Feynman forces on atoms. The Pt (111) surface is modeled using a 3x3 slab model comprising four atomic layers. The top two layers are relaxed, and the bottom two layers are fixed at the bulk lattice constants, which are obtained with the respective functionals. A vacuum of $18 \AA$ is employed, including the adsorbate-covered surface for avoiding any spurious interactions between the periodic images. Dipole corrections are applied to reduce the asymmetry imparted by adsorption at one side of the slab. Gaussian smearing of $0.2 \mathrm{eV}$ is applied, and the energies were extrapolated to zero electronic temperature. The implicit solvation model is applied by using VASPsol with a dielectric constant of 78.4 for water solvent. ${ }^{53,54}$ The explicit model (details given in the solvent model section) has also been used to mimic the solvent-intermediate interactions. Brillouin zone sampling of the slab model is achieved using 3x3 x1 k-point and a Gamma point is used for molecules in a cubic box

of dimension $12 \AA$. The k-point mesh, plane wave energy cutoff and number of atomic layers are verified for convergence. Spin-polarized calculations are carried out for studies involving 
molecular oxygen. The activation barriers are calculated using Climbing-Image Nudged elastic band method (CI-NEB). ${ }^{55}$

\section{Results and Discussions}

\subsection{Structural Details of Catalyst}

The structure-related properties of Pt (111) surface estimated using the considered DFT methods are given in Figure 1. Optimized bulk lattice constants obtained using different methods are compared with the experimental lattice constant of $3.924 \AA$ (Figure 1a). Methods within the same theoretical framework led to differences in lattice constants, as seen for DFT-D2 vs. D3. Applying dispersion correction via the DFT-D3 method shifts the lattice constants towards a better agreement with the experiment for both PBE and RPBE functionals. In contrast, the D2 method underestimates the lattice constant. The highest overestimation of lattice constants by revPBE and rPW86 methods can be expected of arising from the high repulsive interactions offered by these functionals at shorter intermetallic distances, which is in agreement with previous reports for $\operatorname{Pt}(111)$ surface. ${ }^{45}$
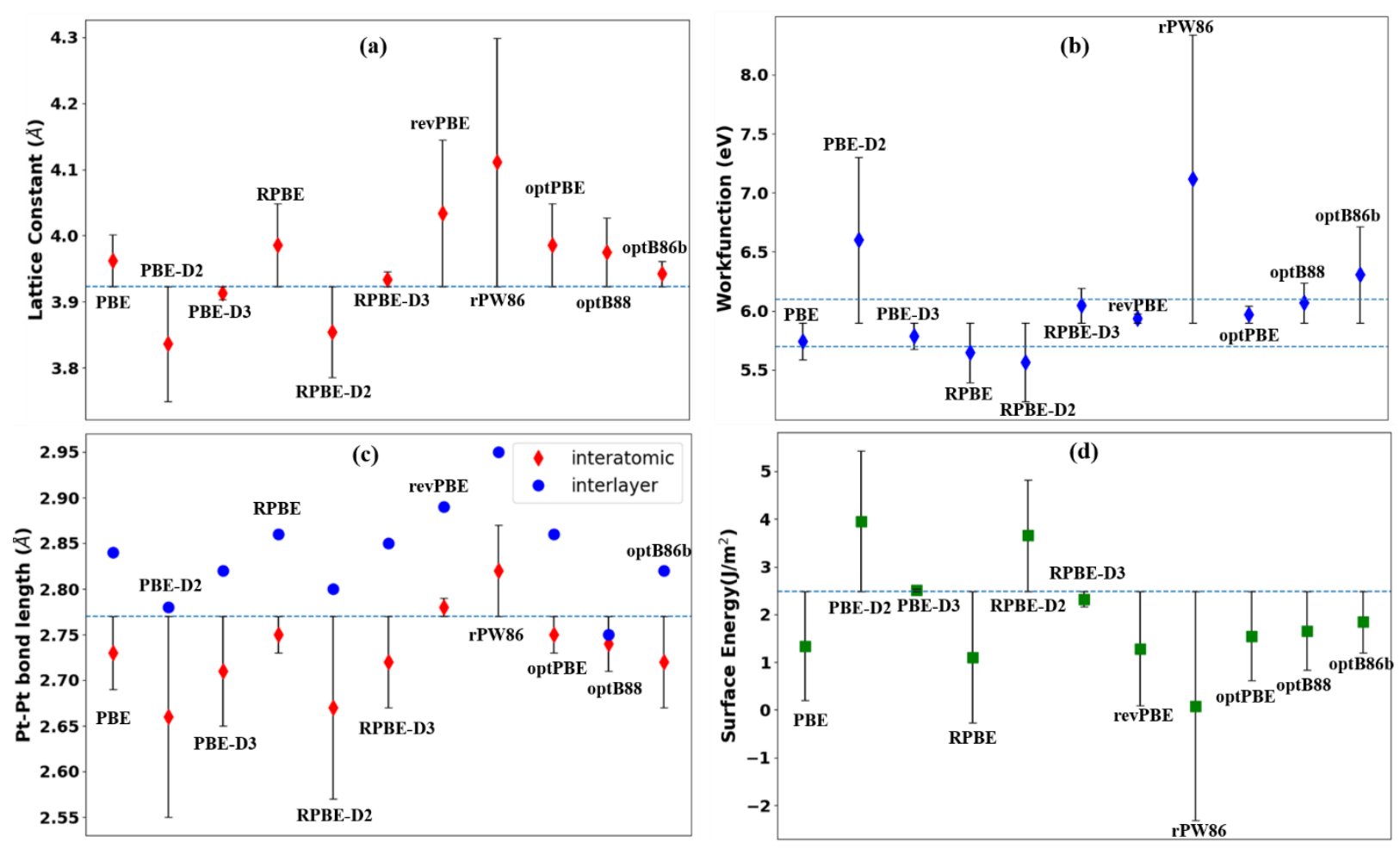
Figure 1: (a) Lattice constant, (b) work function, (c) Pt-Pt interatomic and interlayer bond lengths, and (d) surface energy of $\operatorname{Pt}(111)$ surface calculated using different DFT methods. The error bars are plotted with respect to the experimental value/range from references $45,56-58$, represented by the dashed horizontal line.

A similar overall trend is also observed for $\mathrm{Pt}(111)$ surface work function determined by these methods (Figure 1b). All the methods except PBE-D2 and rPW86 predict a work function value lying close to the experimental range of $5.7-6.1 \mathrm{eV} .^{56,57}$ The structural relaxations of $\mathrm{Pt}$ (111) surface with different methods are quantified in terms of Pt-Pt interatomic distance across the surface and between two relaxed surface layers as shown in Figure 1c. The proximity of Pt-Pt distance observed for methods with the same theoretical framework (PBE-D2; RPBE-D2 and PBE-D3; RPBE-D3) also follows the same trend with experimental Pt-Pt interatomic distance of $2.77 \AA .{ }^{57}$ The standard deviation of interatomic as well as interlayer distance is $\sim 0.05 \AA$, suggesting that reasonable catalytic property differences, if observed any, would not be principally derived from the vastly differing structural predictions between different functionals. Instead, the varying treatment of dispersion interactions within the catalyst-intermediate interface could be more decisive. We have calculated surface energies of the slab models with each method and found that PBE-D3 and RPBE-D3 predict the surface energies closest to the experimental value of $2.49 \mathrm{~J} / \mathrm{m}^{2}$ which can be explained by their fair prediction of bulk lattice constants. ${ }^{58}$ As in the other structurerelated parameters, the rPW86 method highly underestimates the surface energy values (Figure 1d). In general, DFT-D3 and optimized vdW-DF methods are found to predict the $\operatorname{Pt}(111)$ structural features in fair agreement with the experiment.

\subsection{Water Monomer Binding}

Now, we have investigated the water monomer binding energy with different methods, and the results are represented in Figure 2a-b. We have tried three different configurations; i) oxygen atom oriented towards the surface, ii) hydrogen atoms oriented towards the surface, and iii) both oxygen and hydrogen atoms parallel to the surface, as shown in Figure 2a. Water is consistently found to be adsorbed in the parallel configuration. This is in agreement with the previous theoretical results obtained by Zhou et al. ${ }^{59}$ However, the strength of adsorption varies significantly across the methods (0.04-0.6 eV). It can be seen that D2, D3 methods, when used with both PBE and RPBE functionals, significantly improve the monomer binding energies, which is followed by optPBE 
$(0.38 \mathrm{eV})$, optB88 $(0.43 \mathrm{eV})$, and optB86b $(0.46 \mathrm{eV})$ methods with reference to the experimentally measured heat of water adsorption of $0.56 \mathrm{eV} .{ }^{60}$ The lowest binding energy observed for RPBE functional can be correlated with the highest Pt-O bond length of $2.82 \AA$. The higher accuracy of PBE-D3 in predicting water monomer binding energy compared to PBE-D2, which overestimates the $\mathrm{Pt}-\mathrm{H}_{2} \mathrm{O}$ interaction, could be explained by the many-body term involved in the latter.

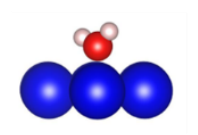

O-down

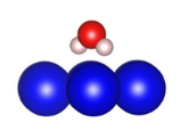

H-down

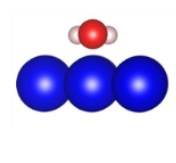

Parallel

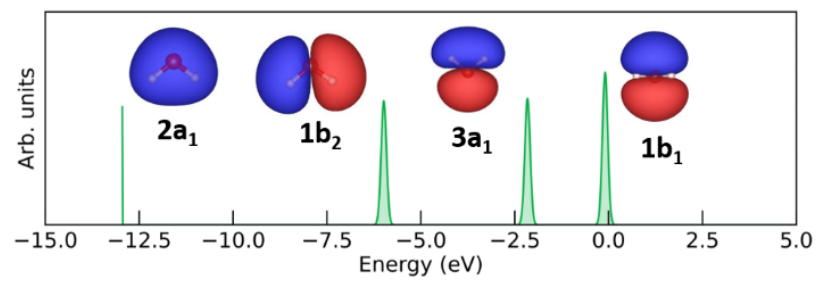

(a)

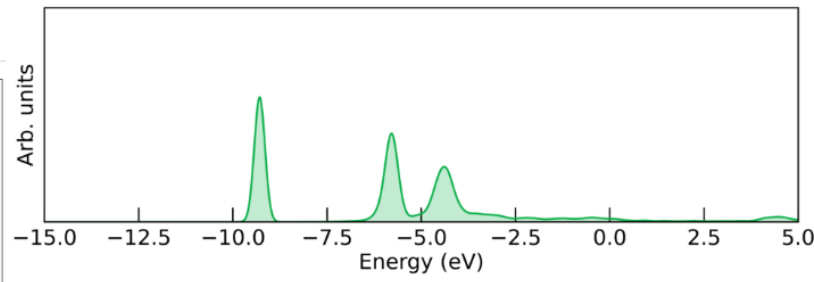

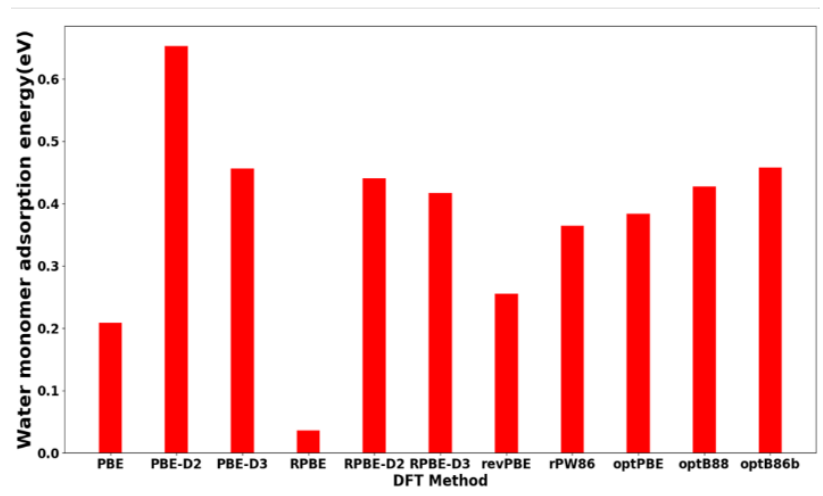

(b)

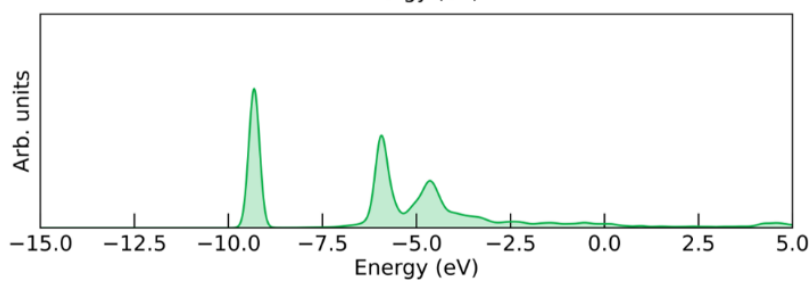

(c)

Figure 2: (a) Different configurations for water monomer adsorption on $\mathrm{Pt}(111)$ surface, (b) Water monomer binding energy with different DFT methods, (c) projected density of states for gas phase $\mathrm{H}_{2} \mathrm{O}$ (top panel), $\mathrm{H}_{2} \mathrm{O}$ adsorbed on Pt(111) with PBE method (middle panel) and PBE-D2 method (bottom panel). The $\mathrm{H}_{2} \mathrm{O}$ molecular orbitals interacting with the surface are labeled in the gas phase diagram.

An investigation into the electronic structure-based nature of the interaction has been carried out by taking PBE and PBE-D2 methods as these methods exhibit the highest disparity between the water monomer binding energies (Figure $2 b$ ) for the same exchange functional. This disparity can be expected to be originating from the $\mathrm{Pt}-\mathrm{H}_{2} \mathrm{O}$ bond length differences of $0.35 \AA$ between these methods. To examine the role of electronic structure in this metal-water interaction, we have conducted density of states (DOS) analysis. The density of states of $\mathrm{H}_{2} \mathrm{O}$ in the gas phase and $\mathrm{H}_{2} \mathrm{O}$ interacting with $\mathrm{Pt}(111)$ surface projected along O-p orbitals at PBE and PBE-D2 level are 
shown in Figure 2c. The metal- $\mathrm{H}_{2} \mathrm{O}$ interaction is mediated primarily by $1 \mathrm{~b}_{1}$ and $3 \mathrm{a}_{1}$ molecular orbitals, which undergo significant broadening while adsorbed on the surface. ${ }^{61}$ The similar energy levels at which the hybridized $\mathrm{Pt}(\mathrm{d})-3 \mathrm{a}_{1}$ and $\mathrm{Pt}(\mathrm{d})-1 \mathrm{~b}_{1}$ states are distributed for PBE and PBE-D2 methods imply that the different $\mathrm{Pt}-\mathrm{H}_{2} \mathrm{O}$ bonding strength predicted by these methods mainly originate from the differences in the treatment of weak-interactions rather than changes in the electronic structure. It is worth mentioning that apart from the role of solvent, water plays a direct role in ORR being the final product along the $4 \mathrm{e}^{-}$reduction pathway. Therefore, the different strength of binding observed for water monomer here is an immediate indication of varying ORR activity among the other methods when water desorption from the metal surface becomes a kinetically dominating process.

\subsection{Solvation Model for ORR}

The solvation effect is an imperative but often overlooked factor for the accurate simulation of heterogeneous catalytic reactions. Though attempts to develop and standardize solvation models for periodic metal surface-based catalysts have been made, a large number of theoretical studies in this direction are still based on gas phase calculations. For an electrocatalytic reaction like ORR, the dynamics of the electrode-electrolyte medium can be important in determining the reaction rate. Liu et al. have reported via a kinetic Monte Carlo (KMC) study that considering water solvent in the chemisorbed state is essential for the accurate prediction of ORR kinetics. ${ }^{39}$ Nonetheless, an empirical approximation based on the stabilization of ORR intermediates in the presence of water molecules has been adapted in many studies, resulting in satisfactory ORR activity prediction from a thermodynamic perspective. ${ }^{62,63}$ Despite the observed validity of such correction methods for predicting activity trends, they do not consider a realistic description of solvent effects. In addition, the plausible DFT-functional sensitivity towards such correction factors is not well calibrated. Recently, Zhang and Ashtagiri reported the inadequacy of such solvent correction methods even after combining with implicit modeling compatible for determining the ORR activity correctly. ${ }^{34}$ 


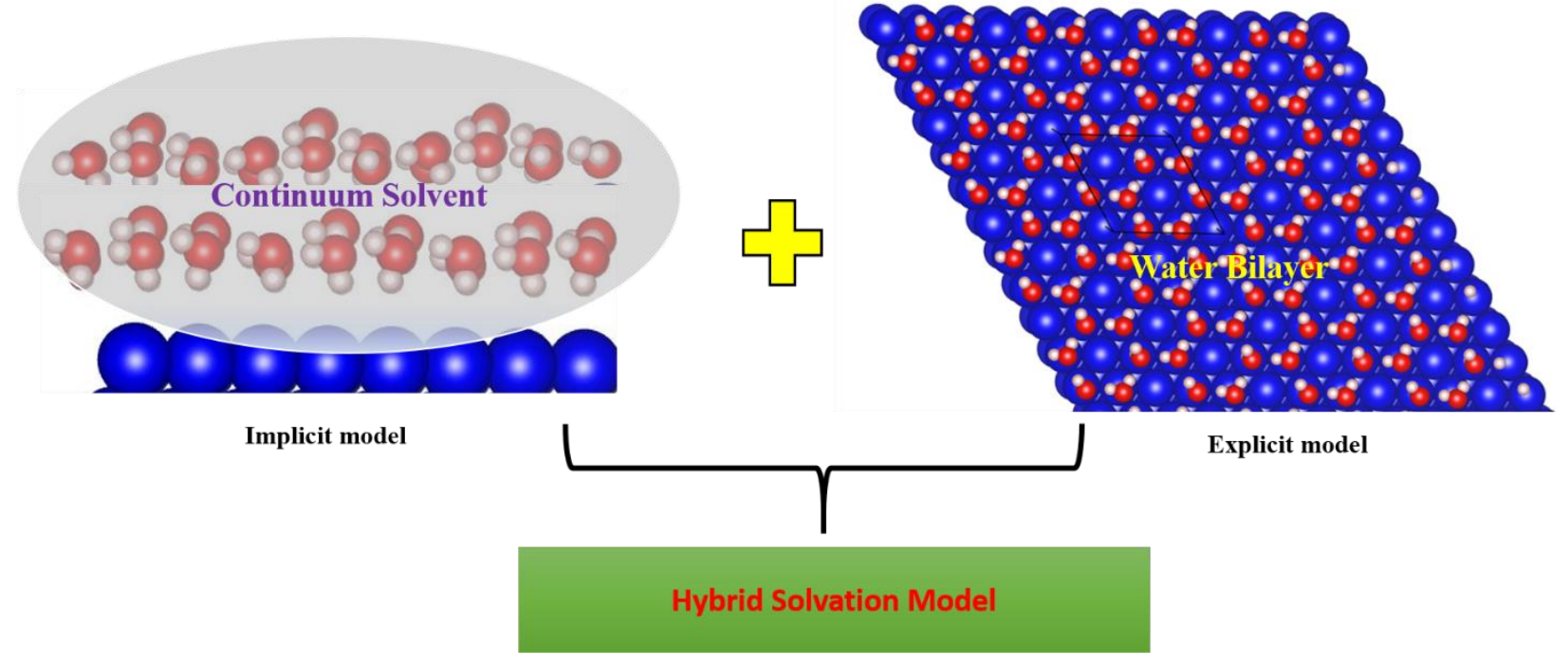

Scheme 1: Schematic representation of hybrid solvation model considered in this study.

The structure and orientation of water molecules on Pt (111) surface has been a focus of intensive research both in experimental and theoretical scenarios. Low energy electron diffraction (LEED) studies have spotted a water bilayer- a hexagonal hydrogen-bonded network with water molecules oriented in two different planes. ${ }^{64}$ This has given rise to the well-known 'bilayer (BL) model' comprising hydrogen-bonded ice-like hexagonal networks of water forming a periodic array over the surface, which has been employed for the theoretical simulations of the electrode-electrolyte interface of Pt (111) surface. ${ }^{65}$ Though some other networks which consist of water pentagons, heptagons have also been experimentally identified, the bilayer model is a more standardized approach and primarily used in understanding the explicit solvation effects for ORR. ${ }^{66,67}$ In our study, we employ a hybrid model, i.e., a combination of an explicit model with water bilayer and an implicit model with a continuous dielectric medium for the ORR simulation (Scheme 1). Predominantly, there are two water bilayer configurations, H-up, in which hydrogen atoms of three alternative water molecules are orientated perpendicular away from the surface and $\mathrm{H}$-down, in which these hydrogen atoms are pointed towards the surface (Figure 3a). In both the configurations, remaining three water molecules have been considered to be parallel to the surface. We have investigated the relative stability between $\mathrm{H}$-up and $\mathrm{H}$-down configurations for all the methods, and the results are represented in Figure S1. It can be seen that except rPW86, all other methods show higher stability for H-down configurations. Different initial geometries were chosen for the water bilayers and selected the stable configurations for further calculations. The adsorbed 
water bilayer differs between functionals in terms of the spatial distribution of water molecules within the hexagonal bilayer (Figure S2). The difference in the extent of interactions of water bilayers is quantified in terms of mean absolute deviation in their bond lengths to the surface and the change in the work function of the surface when water bilayers are included, which are given in Figure S3. On the contrary to the similar configuration observed for water monomer adsorption, the water molecules within the bilayers are in perfect bilayer arrangement from the surface only for RPBE-D3 H-down and rPW86 H-up configurations. This can be understood from the highest average $\mathrm{Pt}-\mathrm{H}_{2} \mathrm{O}$ bond lengths for the BLs in these methods, allowing the inter-molecular interactions to result in a nearly perfect buckled hexagonal network. Nonetheless, the hydrogenbonded network is identified to be retained for all the methods. To understand the stability variation of bilayers over the $\mathrm{Pt}(111)$ surface, the average adsorption energies of the water bilayers are calculated as,

$$
\mathrm{E}_{\mathrm{ads}}=\frac{\left(\mathrm{E}_{\mathrm{Pt}}+\mathrm{n} \times \mathrm{E}_{\mathrm{H}_{2} \mathrm{O}}\right)-\mathrm{E}_{\mathrm{Pt}-\mathrm{H}_{2} \mathrm{O}}}{\mathrm{n}}
$$

where $E_{P t}$ is the total energy of the bare surface, $E_{H_{2} \mathrm{O}}$ is the total energy of the water molecule, $\mathrm{E}_{\mathrm{Pt}-\mathrm{H}_{2} \mathrm{O}}$ is the total energy of the bilayer adsorbed surface system, and $\mathrm{n}$ is the number of water molecules in the unit cell. The calculated adsorption energies are given in Figure $3 \mathrm{~b}$ (blue panel). Including van der Waals interaction significantly improves the adsorption energies of water bilayers over the surface. Both water bilayers and monomers follow a similar trend in adsorption strength across the functionals considered. Hence, the trends in the prediction of metal- $\mathrm{H}_{2} \mathrm{O}$ interaction strength by these methods can be considered to be not affected by the coverage of $\mathrm{H}_{2} \mathrm{O}$ molecules.

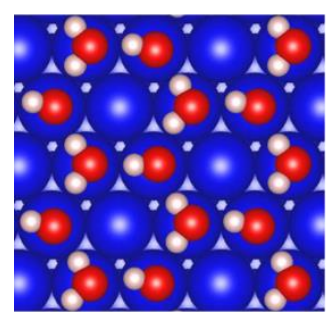

H-down

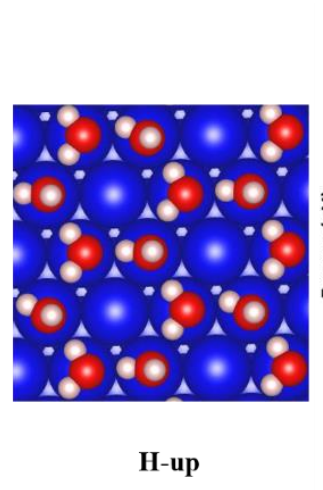

(a)

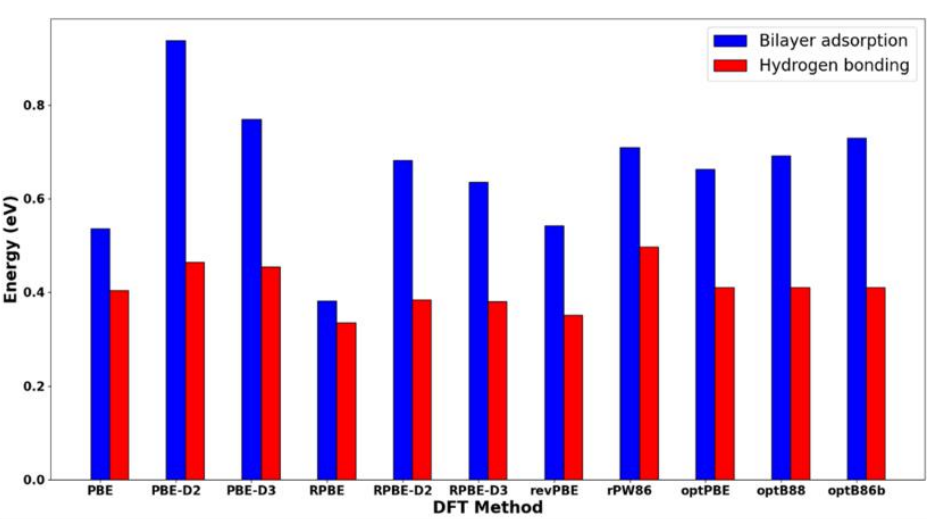

(b) 
Figure 3: (a) H-down and H-up configurations of water bilayer on $\mathrm{Pt}(111)$ surface, (b) Comparison between water bilayer adsorption and inter- $\mathrm{H}_{2} \mathrm{O}$ hydrogen bonding energetics. Blue, red, pink colors represent Platinum, Oxygen, and Hydrogen atoms, respectively. This color code is used throughout the manuscript unless stated otherwise.

Bilayer interaction with the metal surface is a cumulative effect of both metal-water and waterwater interactions. Hence, in order to decouple these interactions, we have calculated the hydrogen bonding energy as;

$$
\mathrm{E}_{\mathrm{HB}}=\frac{\mathrm{E}_{\mathrm{H}_{2} \mathrm{O}}(\text { monomer }) \times \mathrm{N}-\mathrm{E}_{\mathrm{H}_{2} \mathrm{O}}(\mathrm{BL})}{\mathrm{N}}
$$

where, $\mathrm{E}_{\mathrm{H}_{2} \mathrm{O}}$ (monomer) is the total energy of water monomer on the surface, $\mathrm{N}$ is the number of water molecules, $\mathrm{E}_{\mathrm{H}_{2} \mathrm{O}}(\mathrm{BL})$ is the energy of water bilayer in the optimized geometry of the Ptbilayer system. Figure 3(b) shows that all the methods predict a stronger $\mathrm{Pt}-\mathrm{H}_{2} \mathrm{O}$ interaction than the hydrogen bonding within the water bilayer. This hydrophilicity of $\operatorname{Pt}(111)$ surface is previously reported both experimentally as well as theoretically. ${ }^{68,69}$ The overestimation of both the $\mathrm{H}_{2} \mathrm{O}-\mathrm{H}_{2} \mathrm{O}$ and $\mathrm{Pt}-\mathrm{H}_{2} \mathrm{O}$ interactions by the PBE functional compared to RPBE evidenced in our study is reconcilable with the observation of Tongold and Groß. ${ }^{70}$ This trend is followed by their semiempirical dispersion analogs as well. Furthermore, the deviations in hydrogen bonding energy between the methods are less than $\mathrm{Pt}-\mathrm{H}_{2} \mathrm{O}$ binding energy, which is evident from the differences in treating the metal- $\mathrm{H}_{2} \mathrm{O}$ weak interaction.

\subsection{ORR Activity Analysis}

The ORR activity simulation is initiated by analyzing the adsorption characteristics of principal ORR intermediates $\mathrm{O}_{2}, \mathrm{O}, \mathrm{OH}$, and $\mathrm{OOH}$ and $\mathrm{H}_{2} \mathrm{O}$ with the different DFT methods. Adsorption stability analysis of these intermediates carried out in the gas phase shows that the most stable sites for the intermediates do not change with different methods. The obtained stable sites are di-sigma, 3-fold fcc, top, bridge for $\mathrm{O}_{2}, \mathrm{O}, \mathrm{OH}$, and $\mathrm{OOH}$ species. The kinetic stability of the adsorption sites evaluated via the barrier for diffusion of easily mobile intermediate $* \mathrm{O}$ (* indicating adsorbed state) from most stable fcc to next highest stable hcp site shows a nearly constant value of $\sim 0.45$ $\mathrm{eV}$ for all the methods except for rPW86 with a value of $0.37 \mathrm{eV}$ (Figure S4). This designates that the intermediates can be considered to be kinetically trapped in their most stable sites for a 
reasonable timescale. Next, we have considered the adsorption of ORR intermediates within the bilayer model, a representative model of which determined with the PBE method is given in Figure 4. It can be seen that the structural transformations within the water bilayer network are nonuniform across different functionals, as represented in Table S1. The replacement of one of the $\mathrm{H}_{2} \mathrm{O}$ molecules from the water bilayer induces significant structural changes within the BL network. The adsorption of $\mathrm{O}_{2}$ and $\mathrm{O}$ induces the largest structural deviations as the number of hydrogen bonds lost due to the loss of one water molecule is only partially regained. In contrast, $\mathrm{OH}$ and $\mathrm{OOH}$ adsorption has been found to shift the water BL network closer towards perfect bilayer arrangement for all the functionals considered. This can be attributed to the ability of $* \mathrm{OH}$ and $* \mathrm{OOH}$ to reestablish the lost hydrogen bonds. This is also evident from the less hydrogen bonding distance $(\sim 1.5 \AA)$ observed for $* \mathrm{OH}-\mathrm{H}_{2} \mathrm{O}$ and $* \mathrm{OOH}-\mathrm{H}_{2} \mathrm{O}$ interactions for all the functionals compared to higher bond lengths seen for $* \mathrm{O}_{2}$ and $* \mathrm{O}$.
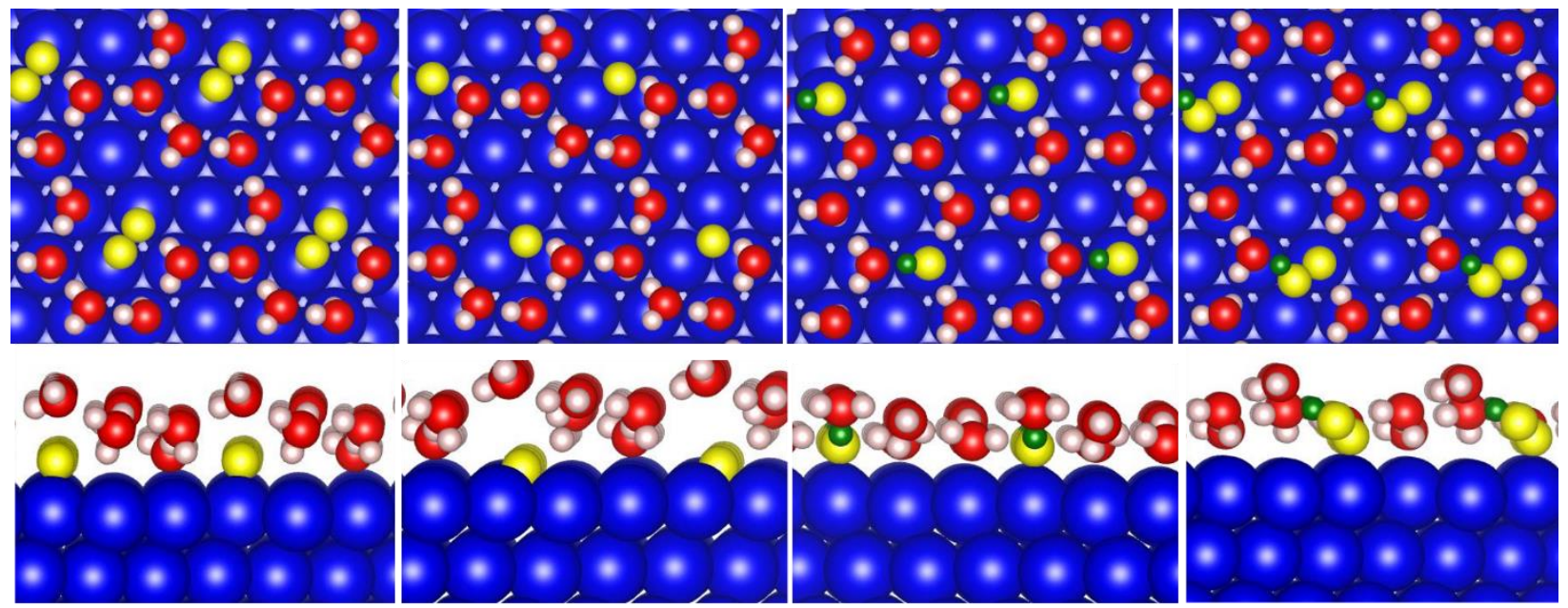

$* \mathrm{O}_{2}$

$* \mathbf{O}$

${ }^{*} \mathrm{OH}$

$* \mathbf{O O H}$

Figure 4: Adsorption configurations (top and side views) of $* \mathrm{O}_{2}$, $* \mathrm{O}, * \mathrm{OH}$, and $* \mathrm{OOH}$ intermediates simulated using the hybrid solvation model. The configurations with the PBE method are shown as a representative model. The oxygen and hydrogen atoms of the ORR intermediate are given yellow and green colors, respectively, to distinguish from the similar atoms in the water BL network.

In order to understand the energetics of adsorption, we have calculated the adsorption free energies of ORR intermediates in both gas phases and solvent phases by following the equations; 


\section{Gas phase:}

$$
\begin{aligned}
& *+2 \mathrm{H}_{2} \mathrm{O}_{(\mathrm{l})} \rightarrow * \mathrm{O}_{2}+4\left(\mathrm{H}^{+}+\mathrm{e}^{-}\right) \\
& *+\mathrm{H}_{2} \mathrm{O}_{(\mathrm{l})} \rightarrow * \mathrm{O}+2\left(\mathrm{H}^{+}+\mathrm{e}^{-}\right) \\
& *+\mathrm{H}_{2} \mathrm{O}_{(\mathrm{l})} \rightarrow * \mathrm{OH}+\left(\mathrm{H}^{+}+\mathrm{e}^{-}\right) \\
& *+2 \mathrm{H}_{2} \mathrm{O}_{(\mathrm{l})} \rightarrow * 0 \mathrm{OH}+3\left(\mathrm{H}^{+}+\mathrm{e}^{-}\right)
\end{aligned}
$$

\section{Solvent Phase:}

$$
\begin{gathered}
* 6 \mathrm{H}_{2} \mathrm{O}+\mathrm{H}_{2} \mathrm{O}_{(\mathrm{l})} \rightarrow * 5 \mathrm{H}_{2} \mathrm{O}-* \mathrm{O}_{2}+4\left(\mathrm{H}^{+}+\mathrm{e}^{-}\right) \\
* 6 \mathrm{H}_{2} \mathrm{O} \rightarrow{ }^{*} 5 \mathrm{H}_{2} \mathrm{O}-* \mathrm{O}+2\left(\mathrm{H}^{+}+\mathrm{e}^{-}\right) \\
* 6 \mathrm{H}_{2} \mathrm{O} \rightarrow * 5 \mathrm{H}_{2} \mathrm{O}-* \mathrm{OH}+\left(\mathrm{H}^{+}+\mathrm{e}^{-}\right) \\
* 6 \mathrm{H}_{2} \mathrm{O}+\mathrm{H}_{2} \mathrm{O}_{(\mathrm{l})} \rightarrow * 5 \mathrm{H}_{2} \mathrm{O}-* 0 \mathrm{OH}+3\left(\mathrm{H}^{+}+\mathrm{e}^{-}\right)
\end{gathered}
$$

From Table 1, it can be seen that the adsorption free energies are shifted to higher values in the solvent phase than gas phase for the majority of functional-intermediate combinations. It is to be noted that here we consider the formation of ORR intermediates on the $\operatorname{Pt}(111)$ surface with respect to the water BL network in contrast to the conventional gas phase scenario where the adsorption energies are calculated with reference to the bare slab model. From the gas phase results, it is evident that the inclusion of dispersion interactions significantly affects the adsorption strength of ORR intermediates on the $\mathrm{Pt}(111)$ surface. All the methods except D2 result in stronger binding of ORR intermediates, whereas the D2 method shows a slightly lesser binding for $*$ O compared to PBE or RPBE methods. The comparatively lesser adsorption feasibility seen in the solvent phase can be expected to be originating from the energy requirement for the structural rearrangements associated with the adsorption of intermediates into the water bilayer. The highest disparity between the adsorption free energies between gas and solvent phases observed for $* \mathrm{O}_{2}$ and $* \mathrm{O}$, which are previously shown to exhibit the maximum structural changes in the BL, supporting this notion. It is noteworthy that the adsorption free energies for $* \mathrm{OH}$ do not differ much between solvent and gas phases as the presence of $* \mathrm{OH}$ causes the hexagonal network to be more rigid. High solvent phase stabilizations are found for $* \mathrm{OH}$ in the case of RPBE-D3 and rPW86 methods 
as they, in the pristine BL form, already exhibit a perfect water-bilayer network and hence increases the stability with a more closed $\mathrm{H}_{2} \mathrm{O}-* \mathrm{OH}$ network offer stabilization. For instance, in the case of rPW86, the water bilayer, which stays in the H-up configuration with an average distance of $3.31 \AA$, the adsorption of $* \mathrm{OH}$ at the top atoms acts as anchoring units between the surface and water bilayer, which provides significant stabilization to them. Other methods such as PBE, RPBE-D3, optB88, and optB86b also are associated with a relatively higher $* \mathrm{OH}$ adsorption strength in the solvent phase. However, ${ }^{*} \mathrm{OOH}$ exhibits a different adsorption behavior with higher adsorption stabilization in the solvent phase observed only for rPW86 and RPBE methods.

Table 1: Adsorption free energies of ORR intermediates with different DFT methods. The corresponding gas phase values are given in parenthesis.

\begin{tabular}{|c|c|c|c|c|}
\hline \multirow{2}{*}{$\begin{array}{c}\text { DFT } \\
\text { Method }\end{array}$} & \multicolumn{4}{|c|}{ Adsorption free energies $(\mathrm{eV})$ of ORR intermediate in solvent (gas) phase } \\
\hline & $* \mathbf{O}_{2}$ & $* \mathbf{O}$ & $* \mathbf{O H}$ & *OOH \\
\hline PBE & $4.69(4.06)$ & $1.70(1.36)$ & $0.80(0.89)$ & $3.91(3.71)$ \\
\hline PBE-D2 & $4.77(3.77)$ & $2.34(1.39)$ & $0.69(0.54)$ & $3.86(3.20)$ \\
\hline PBE-D3 & $4.72(3.84)$ & $2.00(1.30)$ & $0.72(0.69)$ & $3.85(3.39)$ \\
\hline RPBE & $4.96(4.16)$ & $1.71(1.44)$ & $1.08(0.98)$ & $3.78(3.80)$ \\
\hline RPBE-D2 & $4.10(3.87)$ & $2.09(1.49)$ & $0.99(0.68)$ & $3.95(3.34)$ \\
\hline RPBE-D3 & $4.18(3.75)$ & 1.93 & $-0.09(0.73)$ & $3.57(3.31)$ \\
\hline revPBE & $4.14(3.51)$ & $1.56(1.07)$ & $0.77(0.65)$ & $3.47(3.15)$ \\
\hline rPW86 & $3.78(3.31)$ & $1.71(0.88)$ & $-0.37(0.49)$ & $2.18(2.96)$ \\
\hline optPBE & $4.27(3.60)$ & $1.66(1.06)$ & $0.67(0.59)$ & $3.49(3.17)$ \\
\hline optB88 & $4.16(3.59)$ & $1.80(1.02)$ & $0.48(0.57)$ & $3.53(3.21)$ \\
\hline optB86b & $4.26(3.53)$ & $1.74(1.04)$ & $0.58(0.61)$ & $3.46(3.14)$ \\
\hline
\end{tabular}


This can be ascribed to the orientational differences associated with * OOH binding within the BL network across different functionals, which changes the binding strength despite regaining the $\mathrm{H}$ bond network. Also, the trends in the adsorption free energies between different methods are different in the gas phase and solvent phase, significantly affected by dispersion interactions in both gas and solvent phases where the trends can be non-linear between the two phases depending on the choice of the DFT method. For example, when RPBE-D2 increases the *OOH binding compared to RPBE in the gas phase, it shows an opposite trend in the solvent phase. These results suggest that the stability of ORR intermediates on $\operatorname{Pt}(111)$ surface can be highly varying between different methods.

To understand the solvation effect on the stabilization of ORR intermediates, we have calculated the solvation energy for all the methods (Figure 5). The solvation energy is determined as the difference between the free energy of adsorption for an ORR intermediate at the gas phase and solvent phase. It can be understood that the observed adsorption free energy trend is visible on the solvation energy values. The comparatively lesser values for $* \mathrm{OH}$ and $* \mathrm{OOH}$ indicates that the reaction steps mediated by these intermediates can also be affected by solvent effects. The high negative solvation energy for *OH for RPBE-D3 and both $* \mathrm{OH}$ and $* \mathrm{OOH}$ for $\mathrm{rPW} 86$ is a measure of the slightest structural changes imparted by removing $\mathrm{H}_{2} \mathrm{O}$ molecules for the occupation of ORR intermediates, as evident from their structural analysis. Compared to PBE functional, RPBE exhibits largely differing solvation energy trends within the D2, D3 framework associated with it. Within the vdw-DF scenario, the optPBE, optB88, and optB86b show nearly similar solvation energy trends. 


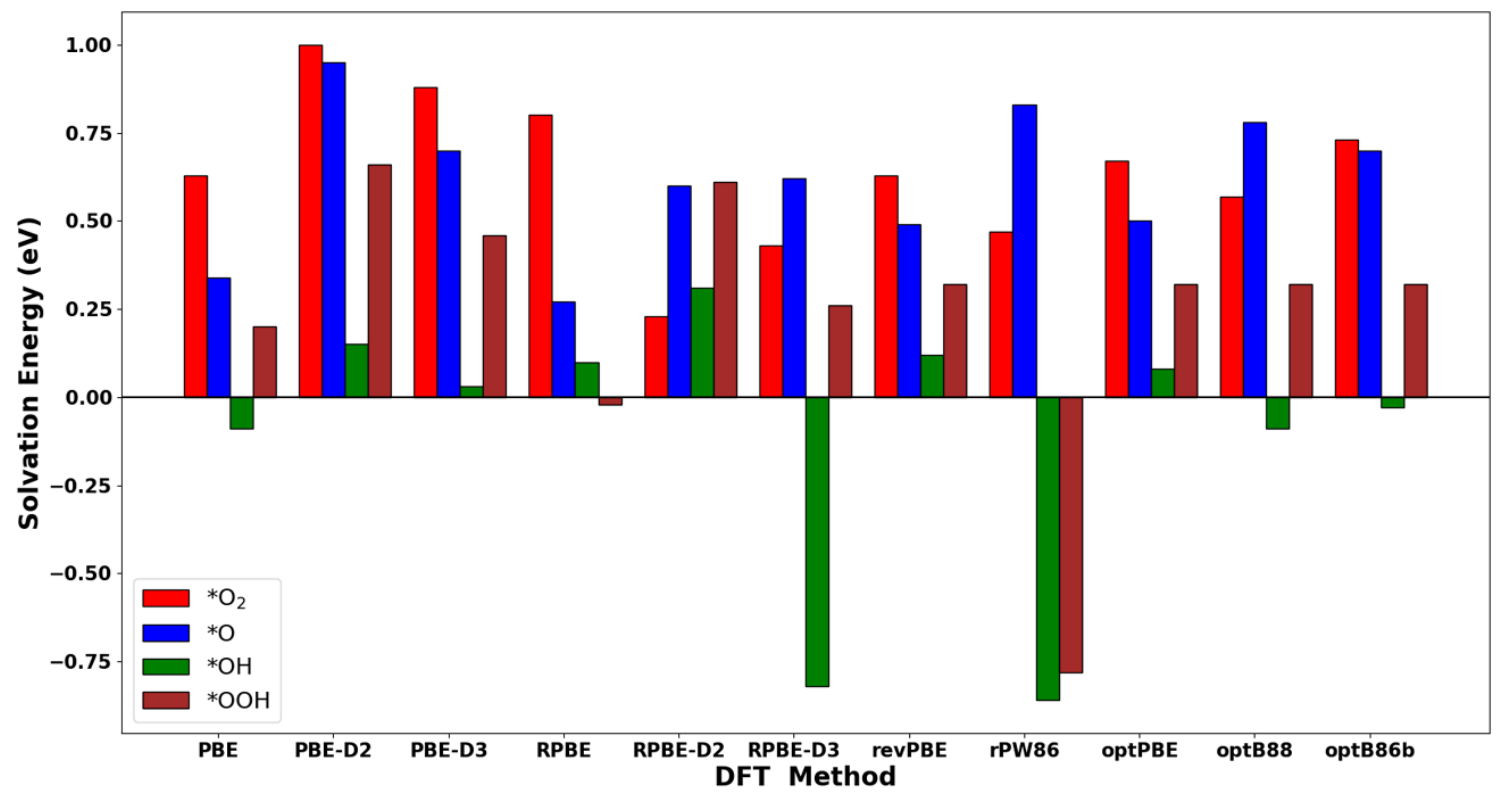

Figure 5: Solvation energy associated with the adsorption of $* \mathrm{O}_{2}$, $* \mathrm{O}, * \mathrm{OH}$, and $* \mathrm{OOH}$ intermediates determined for different DFT methods.

Next, we try to analyze the behavior of scaling relationships between the adsorption free energies of ORR intermediates as these have been largely used for understanding the ORR. Scaling relations have been widely used to understand the origin of activity and predict new catalytic systems with minimal computational efforts. ${ }^{71,72}$ The scaling relations are generally studied over a set of catalyst surfaces where the reported standard relations are $\Delta \mathrm{GO}=\Delta \mathrm{GOH}+2$ for $\mathrm{O}$ vs. $\mathrm{OH}$ and $\Delta \mathrm{GOOH}=\Delta \mathrm{GOH}+3.2$ for $\mathrm{OH}$ vs. $\mathrm{OOH}$ intermediates $\left(\Delta \mathrm{G}\right.$ denoting adsorption free energy). ${ }^{73}$ Here, we attempt to check the plausibility of such a scaling relationship across different DFT methods for the Pt (111) surface. Previously, Norskov and co-workers have reported the sustainability of $\mathrm{OH}$ vs. $\mathrm{OOH}$ scaling relation across different functionals despite the systematic error, which was canceled by the inclusion of van der Waal's corrections. ${ }^{35}$ However, their study had not considered the effect of solvation implicitly and/or explicitly. The obtained scaling relations in both the gas phase and solvent phase for the ORR intermediates for different methods are depicted in Figure 6. It can be seen that there exists a reasonable correlation between $\Delta \mathrm{GOH}$ vs. $\triangle \mathrm{GOOH}$ in both the gas phase and the solvent phase, where it is highest for the former with a coefficient of determination value of 0.92 . In both solvent and gas phases, the $\mathrm{OH}$ and $\mathrm{OOH}$ adsorption free energies are better correlated than $\mathrm{O}$ vs. $\mathrm{OH}$, almost negligible in the solvent phase. 
This can be directly attributed to the significant differences in the structural changes associated with $\mathrm{O}$ and $\mathrm{OH}$ adsorption in the water bilayer network with different methods, offering significantly different stabilizations and hence adsorption free energies. It needs to be noted that the slope and intercept values we observe for these scaling relations are different from the standard values, as here, the dataset is more heterogeneous involving functionals rather than a homogenous dataset involving catalyst surfaces. The lesser correlation obtained in the solvent phase suggests that the errors arising from the description of ORR intermediate interaction with the metal surfaces are more than those found in a gas phase. Therefore, dispersion correction method-dependent errors can be expected in optimizing the catalyst selection from their optimum adsorption free energy based on scaling relations, especially for those systems where the potential limiting step involves $* \mathrm{O}$ to $* \mathrm{OH}$ formation, which is true for many ORR catalysts.

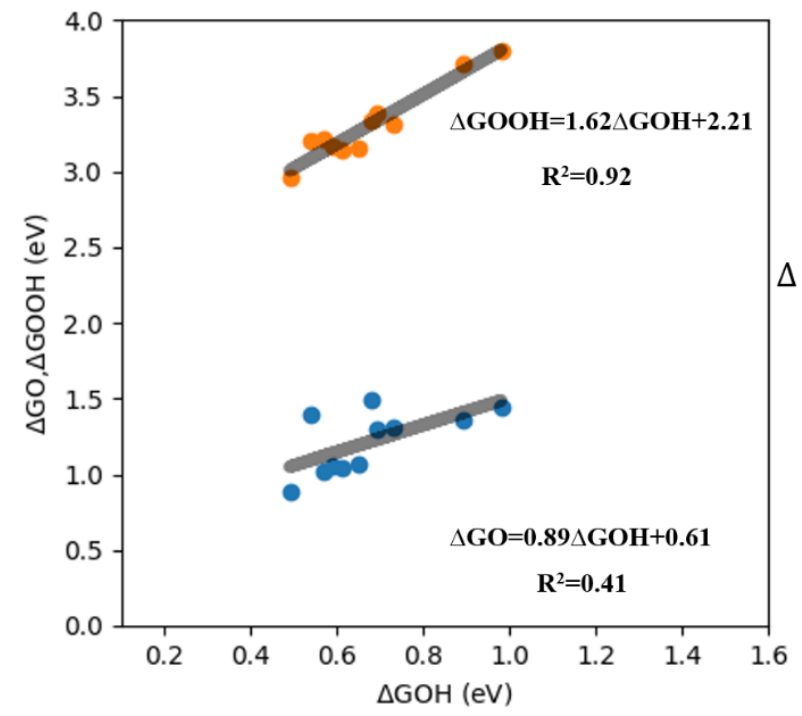

(a)

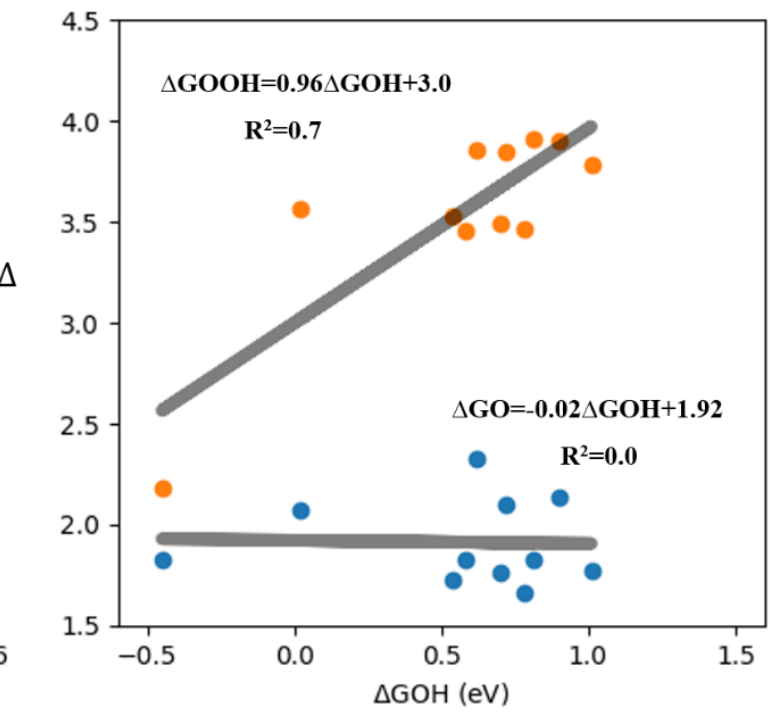

(b)

Figure 6: DFT method dependent $\mathrm{O}$ vs. $\mathrm{OH}$ (blue circles) and $\mathrm{OH}$ vs. $\mathrm{OOH}$ (orange circles) scaling relation of adsorption free energies in (a) gas phase, (b) solvent phase.

We have also analyzed the effect of adsorbate coverages in the catalyst-intermediate interaction by studying the ${ }^{*} \mathrm{OH}$ adsorption energetics at different ${ }^{*} \mathrm{OH}$ coverages. The structural configurations of $* \mathrm{OH}$ coverages of $0.11,0.22$ and $0.33 \mathrm{ML}$ are given in Figure S5, and the corresponding adsorption free energies for different DFT methods are represented in Figure S6. It can be seen that except RPBE-D3, revPBE, and rPW86, all other methods exhibit a weakening of 
*OH adsorption free energy with an increase in coverage. This is expected as changing from 0.11 ML to $0.33 \mathrm{ML}$ transforms the $* \mathrm{OH}$ adsorbed water $\mathrm{BL}$ towards a half-dissociated $* \mathrm{OH}-\mathrm{H}_{2} \mathrm{O}$ network. The higher planarity of such a configuration strengthens the rigidity of the hydrogen bonding network in spite of the overall reduction in the number of hydrogen bonds due to the replacement of $\mathrm{H}_{2} \mathrm{O}$ molecules with $* \mathrm{OH}$. As a result, the intramolecular interactions dominate over the metal-surface interactions, and hence the binding energy decreases. For the RPBE-D3, revPBE, and rPW86 methods, since the low-coverage BL configurations themselves are associated with reasonable planarity, the increment in coverage does not result in a linear decrement in binding energy. Rather, there is competition between metal-surface and intra-bilayer interactions, resulting in abrupt changes in the binding energy.

Since under the ORR relevant working potential $(\sim 0.9 \mathrm{~V})$, the $\operatorname{Pt}(111)$ surface sites have been identified to be occupied by $* \mathrm{OH}$ or $* \mathrm{O}$ species, we attempt to understand the potential dependent coverage effects on the stability of the catalyst system with different DFT methods considered. Since $* \mathrm{O}$ adsorption has been identified to largely disrupting the $\mathrm{BL}$, gas phase coverage simulations are applied for $* \mathrm{O}$. We construct surface Pourbaix diagrams to understand the most stable surface state and the trends in relative stability between different surface states. ${ }^{74,75}$ The considered coverages are 0.11 ML, 0.22 $\mathrm{ML}$ and $0.33 \mathrm{ML}$ for both the $* \mathrm{O}$ and $* \mathrm{OH}$ species. It is to be noted that this does not cover the entire coverage range possible for these intermediates to have on $\operatorname{Pt}(111)$ surface. However, we are constrained to these coverages in tandem with the objective to understand the role of dispersion interactions in predicting the relative stability of different surface states rather than identifying the most stable state precisely with each method. The first order surface Pourbaix diagrams constructed between free energy vs. the applied potential for van der Waal's interaction-excluded and semi-empirical methods are shown in Figure 7(a), and that for vdw-DF methods is given in Figure S7. Similarly, second order Pourbaix diagrams constructed between the applied potential vs. $\mathrm{pH}$ for the van der Waal's interaction-excluded and semi-empirical methods are shown in Figure 7(b), and that of vdw-DF methods is given in Figure S8. It is known that under the potential range of $\sim 0.6-0.8 \mathrm{~V}$, the $\operatorname{Pt}(111)$ surface would be occupied by $* \mathrm{OH}$, which would be replaced via electrooxidation by $* \mathrm{O}$ at higher potentials. ${ }^{74}$ From the figure, it can be understood that in the ORR relevant potential range of $>0.8 \mathrm{~V}$, all the methods predict higher stability for *O occupied $\mathrm{Pt}(111)$ surface. Nonetheless, the relative stability differences between different states have been found to show a method dependency. When no 
dispersion interactions are taken into account, both PBE and RPBE functionals predict nearly a direct transition from bare $\operatorname{Pt}(111)$ surface to $0.33 \mathrm{ML}^{*} \mathrm{O}$ covered surface whereas $0.33 \mathrm{ML} * \mathrm{OH}$ covered surface, which has been found to be stable for a reasonable potential range with D2 and D3 methods. This suggests that the ${ }^{*} \mathrm{OH}$ coverage-dependent interactions are treated differently when dispersion interactions are taken under consideration. The second order Pourbaix diagram also conveys important insights regarding the stability of different surface states under $\mathrm{pH}$ conditions (Figure 7b). PBE and RPBE functionals predict a longer $\mathrm{pH}$ range for bare $\mathrm{Pt}(111)$ surface without dissolution. However, when the dispersion interactions are included, the surface is featured with a lower onset $\mathrm{pH}$ value at which the dissolution initiates owing to the stabilization of ORR intermediates $* \mathrm{O}$ or $* \mathrm{OH}$. Within van der Waals functionals, the highest stabilization is observed for $0.33 \mathrm{ML} * \mathrm{O}$ in agreement with the low adsorption free energy associated with the formation of the next immediate stable $0.33 \mathrm{ML} * \mathrm{OH}$ coverage. Among these, rPW86 shows much earlier oxidation of $\mathrm{Pt}(111)$ surface at a $\mathrm{pH}$ of 6.5 with the formation of $0.33 \mathrm{ML} * \mathrm{OH}$ covered surface along with a higher stable potential window for *OH covered state. Since the most stable surface state under ORR relevant potentials and $\mathrm{pH}$ range are predicted uniformly in spite of the differences in the relative stabilities with different methods, we envision that a common theoretical framework can be employed to analyze the energetics and kinetics of ORR.
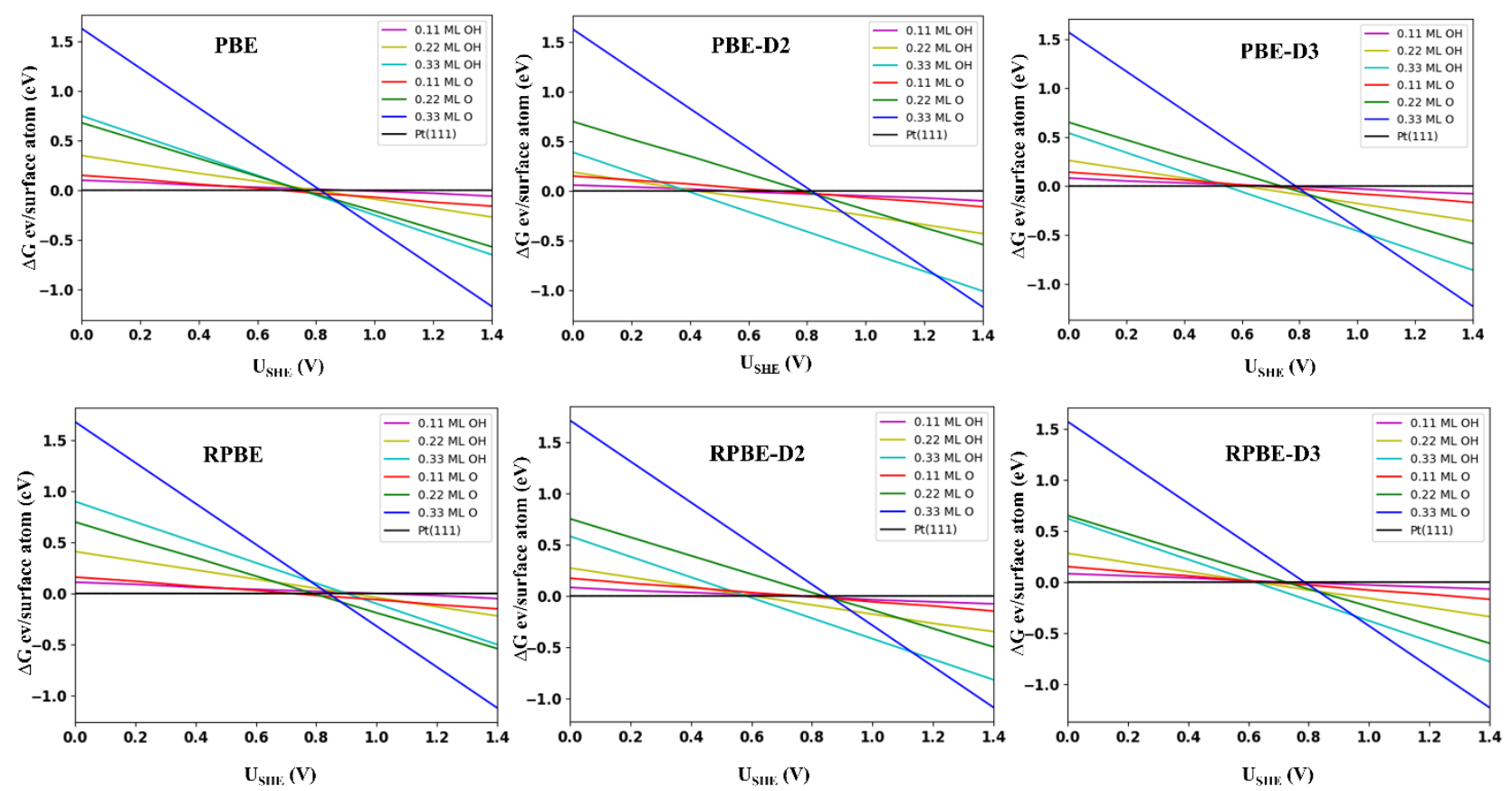

(a) 

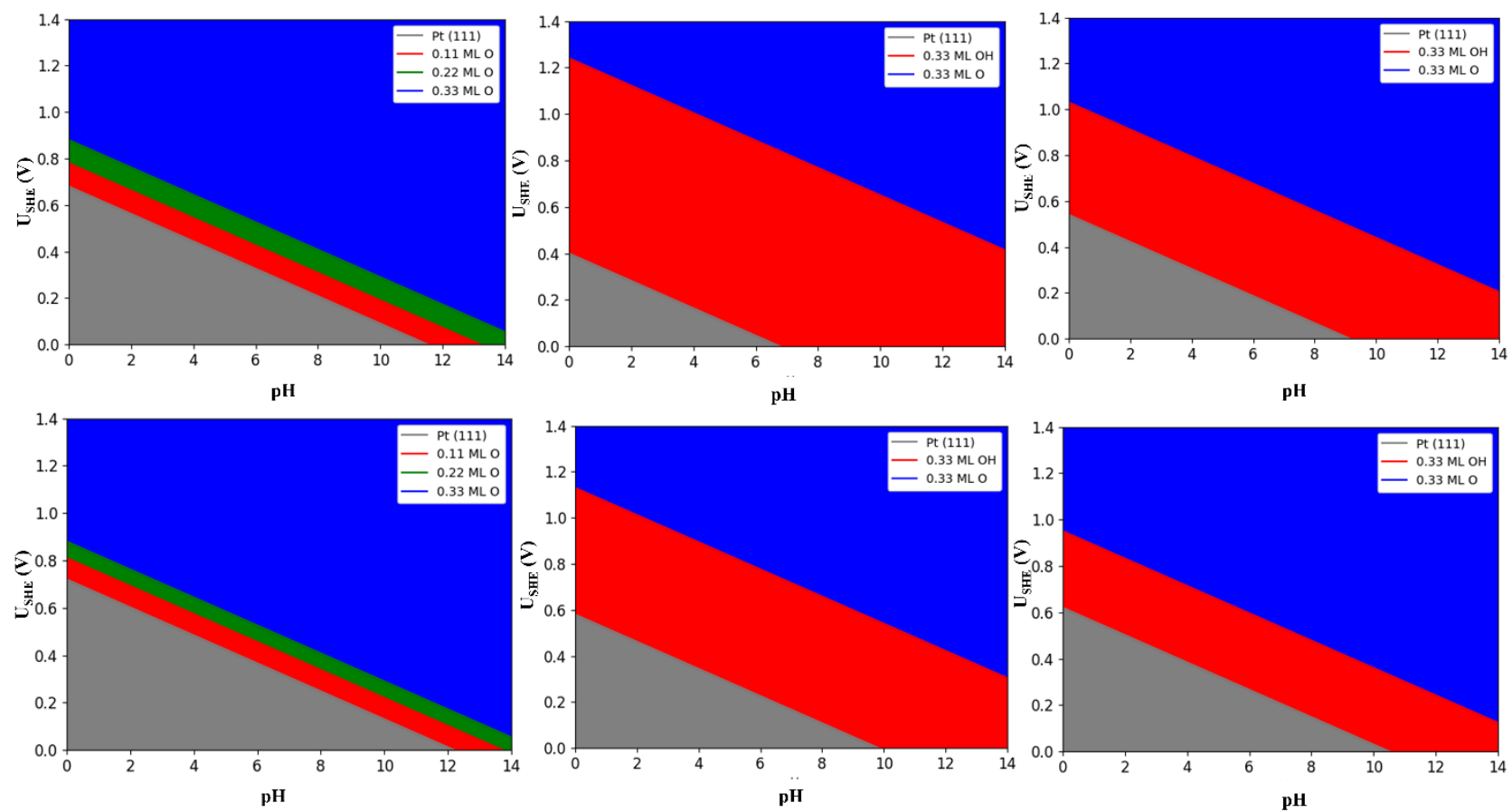

(b)

Figure 7: (a) First order surface Pourbaix diagrams constructed between applied potential and surface free energies, (b) second order surface Pourbaix diagrams constructed between applied potential and $\mathrm{pH}$ range (lower panel) at different $* \mathrm{O}$ and $* \mathrm{OH}$ coverages for non-dispersion as well as semi-empirical dispersion-based methods. The corresponding information for vdw-DF methods is given in Figures S7and S8 of supporting information.

Witnessing the different energetics associated with the adsorption of intermediates predicted by different DFT methods, we attempt to scrutinize the role of dispersion effects in determining the overall ORR activity on $\operatorname{Pt}(111)$ surface. We have considered a reaction network proposed by Hansen et al. ${ }^{76}$ where the intermediates involved in the elementary steps occupy the water adsorbed sites as well as interstitial sites (Scheme 2). These include $* \mathrm{O}_{2} \_\mathrm{S} 1$, *O_S2, ${ }^{*} \mathrm{O} \_\mathrm{S} 2$, $* \mathrm{OH} \_\mathrm{S} 1$, *OH_S2, *OOH_S1, $* \mathrm{H}_{2} \mathrm{O} \_\mathrm{S} 1,{ }^{*} \mathrm{H}_{2} \mathrm{O}_{2} \_\mathrm{S} 1$ where $\mathrm{S} 1$ and $\mathrm{S} 2$ represent the platinum surface site bound by water molecules in the BL and the interstitial sites, respectively. The reaction network includes the diffusion of molecular $\mathrm{O}_{2}$ from bulk electrolyte $\left(\mathrm{O}_{2}(\mathrm{aq})\right)$ to the electrode-electrolyte double layer region at the electrode $\left(\mathrm{O}_{2}(\mathrm{dl})\right)$ followed by adsorption on the catalyst surface $\left(* \mathrm{O}_{2}\right) .{ }^{76}$ From this state, five further pathways are investigated by using the adapted reaction network i) $* \mathrm{OOH}$ reduction pathway in which four-electron reductions leading to the formation of $\mathrm{H}_{2} \mathrm{O}$ involving solemnly electrochemical reduction steps, ii) $* \mathrm{O}_{2}$ dissociation pathway involving four- 
electron reduction forming $\mathrm{H}_{2} \mathrm{O}$ via $\mathrm{O}-\mathrm{O}$ bond dissociation in $* \mathrm{O}_{2}$, iii) $* \mathrm{OOH}$ dissociation pathway involving four-electron reduction forming $\mathrm{H}_{2} \mathrm{O}$ via $\mathrm{O}-\mathrm{O}$ bond dissociation in $* \mathrm{OOH}$, iv) ${ }^{*} \mathrm{H}_{2} \mathrm{O}_{2}$ dissociation pathway involving four-electron reduction forming $\mathrm{H}_{2} \mathrm{O}$ via $\mathrm{O}-\mathrm{O}$ bond dissociation in $* \mathrm{H}_{2} \mathrm{O}_{2}$, v) $\mathrm{H}_{2} \mathrm{O}_{2}$ formation pathway involving two-electron reduction forming $\mathrm{H}_{2} \mathrm{O}_{2}$ via $* O O H$ protonation step. It is to be noted that the ORR pathways differ by the nature of few elementary steps, the products of which follow merging pathways at the final stage. For instance, the *OOH_S1 dissociation step results in *O_S1 co-adsorbed with *OH_S2, which can lead to the formation of either $* \mathrm{OH} \_\mathrm{S} 1+* \mathrm{OH} \_\mathrm{S} 2$ or $* \mathrm{O} \_\mathrm{S} 1+\mathrm{H}_{2} \mathrm{O}$ depending on whether protonation precedes on $* \mathrm{O}$ or $* \mathrm{OH}$. The $* \mathrm{O}$ or $* \mathrm{OH}$ leaving behind the desorption of $\mathrm{H}_{2} \mathrm{O}$ can undergo further protonation, exactly similar to the final steps along the $* \mathrm{OOH}$ electrochemical reduction pathway.

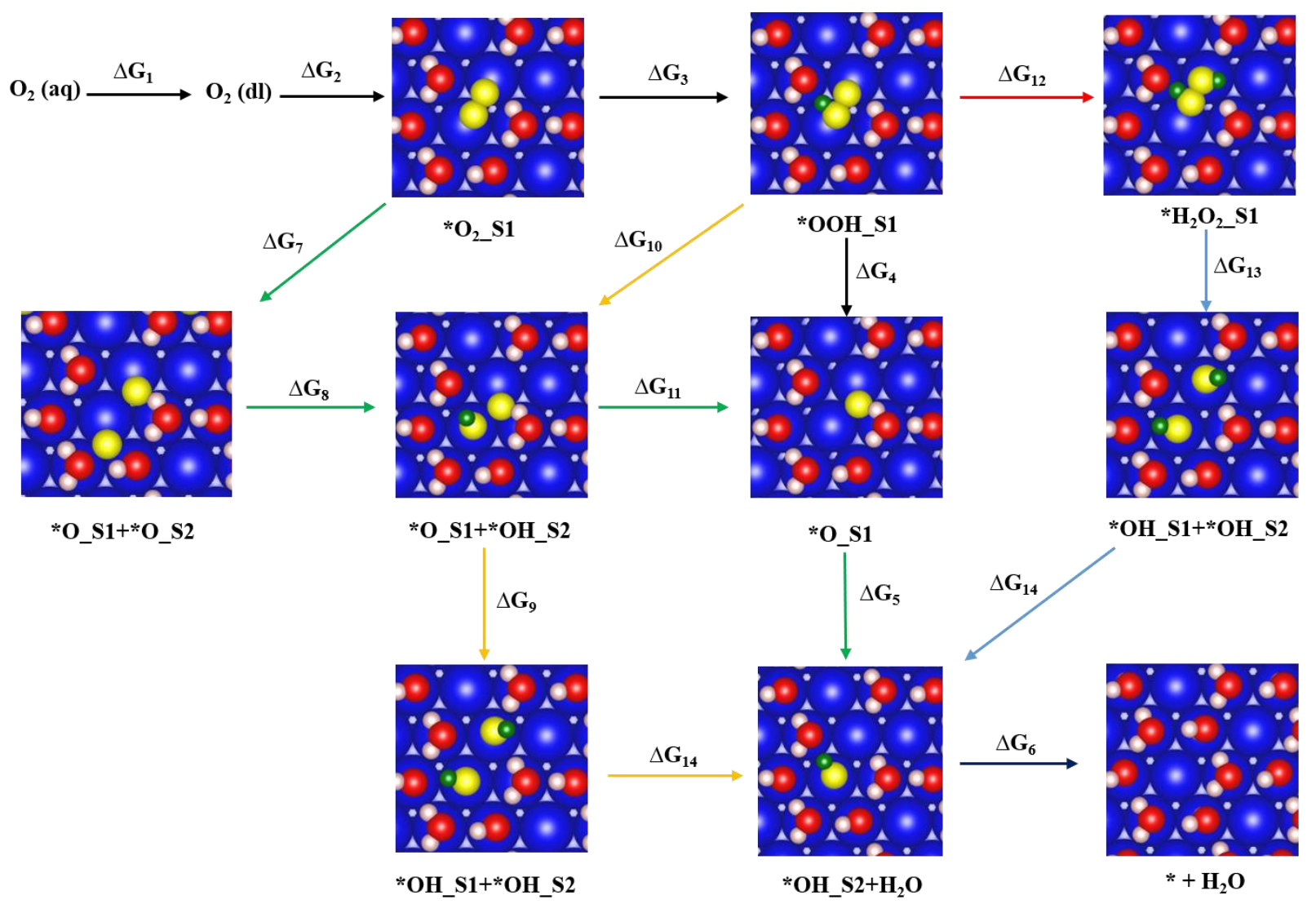

Scheme 2: Reaction network constructed for ORR activity simulation. $\mathrm{S} \_1$ and $\mathrm{S} \_2$ represent $\mathrm{H}_{2} \mathrm{O}$ adsorbed and interstitial sites within the $\mathrm{BL}$ network, respectively. The * $\mathrm{OOH}$ reduction pathway, $\mathrm{O}_{2}$ dissociation pathway, $* \mathrm{OOH}$ dissociation pathway, $\mathrm{H}_{2} \mathrm{O}_{2}$ dissociation pathway, and $\mathrm{H}_{2} \mathrm{O}_{2}$ 
formation pathway are labeled by black, green, orange, blue, and red connections, respectively. For pathways involving similar steps, only unique steps are labeled.

The Gibbs free energy changes $(\Delta \mathrm{G})$ associated with all the elementary steps in the considered reaction network across all the methods is represented as a heat map in Figure 8.

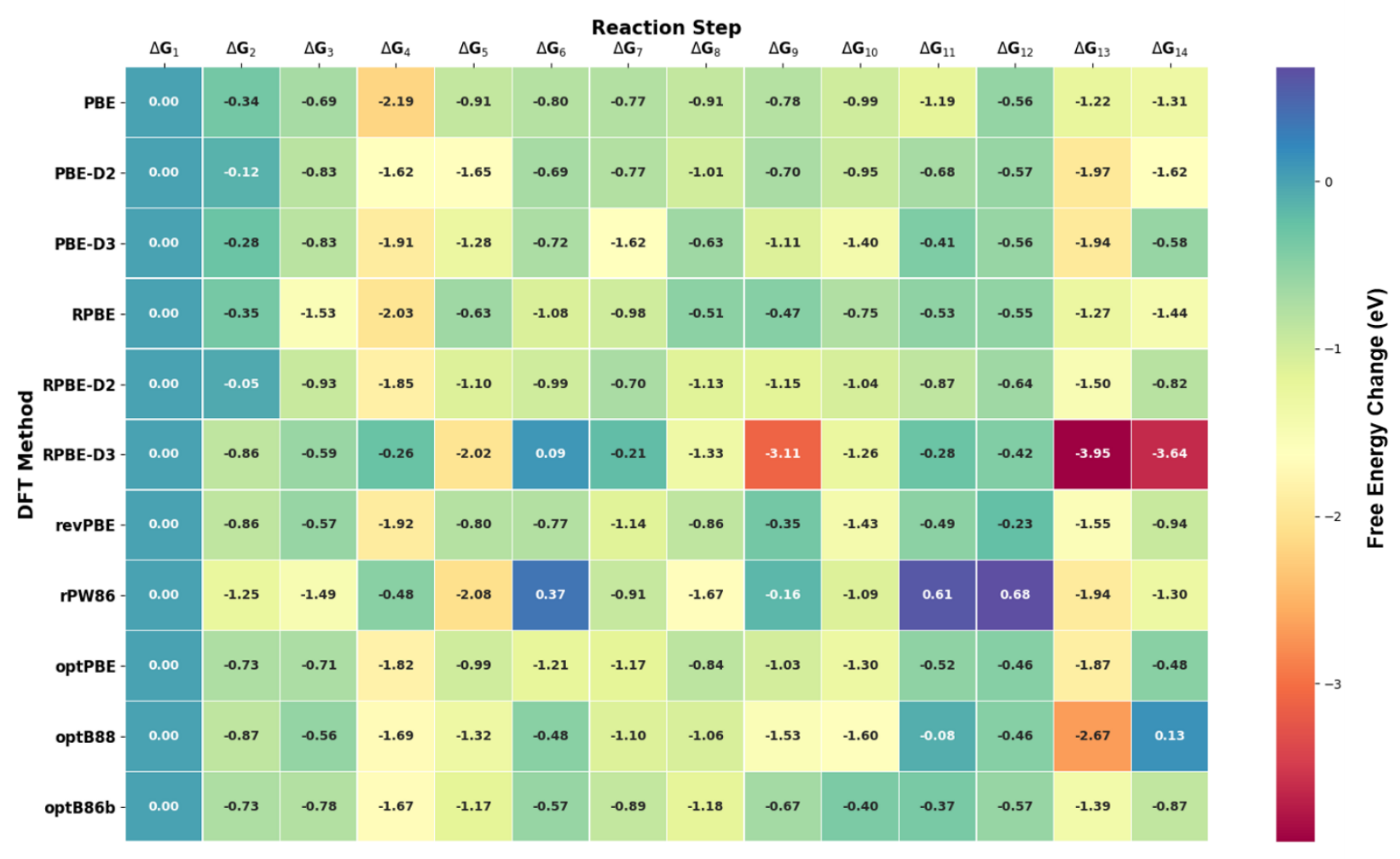

Figure 8: Heatmap representing the variation of ORR energetics across the DFT methods by free energy differences of the corresponding limiting reaction step.

The endergonicity associated with *OH protonation without any applied potential for RPBE-D3 and rPW86 methods can be correlated with the high *OH stabilization via solvation effect identified for these methods. It is to be noted that the notion of limiting step is important for electrochemical steps as they become more energetically uphill when the external potential is applied. In contrast, the $\mathrm{O}_{2}$ adsorption and dissociation steps that are found to be least favorable for PBE, RPBE methods with and without dispersion considerations would not be varying under applied potential. It can be seen that the most facile pathway for all the methods is $* \mathrm{OOH}$ reduction, in which $* \mathrm{OH} \_\mathrm{S} 1 \rightarrow *+\mathrm{H}_{2} \mathrm{O}$ turns out to be the rate-limiting step except for PBE where 
$* \mathrm{O} \_\mathrm{S} 1+* \mathrm{OH} \_\mathrm{S} 1 \rightarrow * \mathrm{OH} \_\mathrm{S} 1+* \mathrm{OH} \_\mathrm{S} 2$ is the rate-limiting step. The congruence of the majority of DFT methods predicting $* \mathrm{OH}$ protonation as the limiting step with the experimental report is a noteworthy observation. ${ }^{77}$ The same step is also found as the rate-limiting step in all the considered pathways for the RPBE-D3 method but associated with a higher thermodynamic energy barrier. The rate-limiting step and limiting potentials along each pathway are enlisted in Table S2. The less thermodynamic feasibility associated with $* \mathrm{OH}$ hydrogenation in the presence of co-adsorbed $* \mathrm{O}$ or $* \mathrm{OH}$ for many methods implies a stronger binding compared to only $* \mathrm{OH}$ along the $* \mathrm{OOH}$ electrochemical reduction pathway. This is expected as the occupancy of the co-adsorbate at the interstitial site acts as an anchoring unit, increasing the binding of the reactive intermediate undergoing protonation and hence resulting in lesser feasibility. It has been well-known that two important competing steps for ORR are $* \mathrm{O}_{2}+* \mathrm{H} \rightarrow * \mathrm{OOH}$ vs. ${ }^{*} \mathrm{O}_{2} \rightarrow * \mathrm{O}+* \mathrm{O}$, which determines the further pathways followed by the intermediates. ${ }^{78}$ Under no applied potential, it has been found while PBE-D2, RPBE, PBE-D2, RPBE-D3, and rPW86 methods favor $* \mathrm{O}_{2}$ protonation, the rest of the methods favor $* \mathrm{O}_{2}$ dissociation revealing reasonably high specificity of DFT methods towards determining the intermediate steps involved in different pathways. The energetic difference between these two steps is not significant enough (maximum of $0.59 \mathrm{eV}$ for rPW86) so that under bias, $* \mathrm{OOH}$ formation becomes less favorable for all the methods. Furthermore, the protonation of dissociated products from $* \mathrm{O}_{2}$ is still associated with a higher thermodynamic barrier which assigns $* \mathrm{OOH}$ reduction as the most facile pathway for all the methods. From the results, it is evident that semi-empirical dispersion combined GGA functionals predict different thermodynamics compared to bare GGA functionals. The rPW86 method, which has been previously found to poorly describe the interaction with the intermediates as well as the surface properties, is associated with the lowest potential along all the pathways suggesting its least competence in predicting the correct ORR activity. All the methods except RPBE-D3 and optB88 are featured with $* O \mathrm{OH}_{-} \mathrm{S} 1 \rightarrow \mathrm{H}_{2} \mathrm{O}_{2}$ as the limiting step for the $\mathrm{H}_{2} \mathrm{O}_{2}$ dissociation pathway, where optB86b has a competing $* \mathrm{OH}$ reduction step along with this. All the thermochemical reactions such as $* \mathrm{O}_{2}$ dissociation, $* \mathrm{OOH}$ dissociation, and $* \mathrm{H}_{2} \mathrm{O}_{2}$ dissociation are featured with high negative free energies for all the methods. This can be ascribed to the more closed occupation of the dissociated intermediates towards the surface compared to the reactant species located at a higher distance from the surface, being part of the disrupted water network. The onset potentials 
along the most facile reaction pathway for all the methods are plotted in Figure 9a with reference to the experimental value of $0.78 \mathrm{~V} .^{79}$
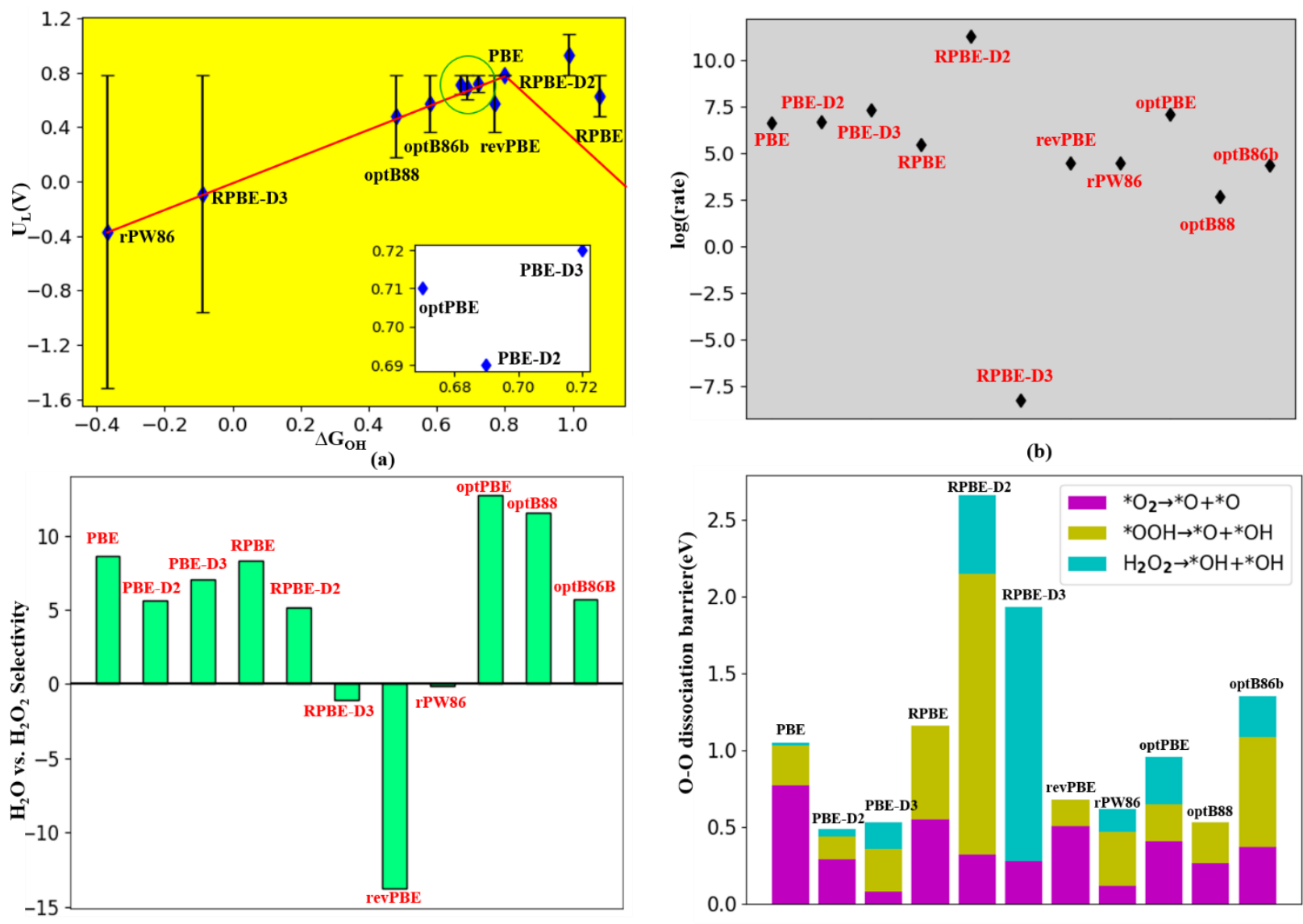

(c)

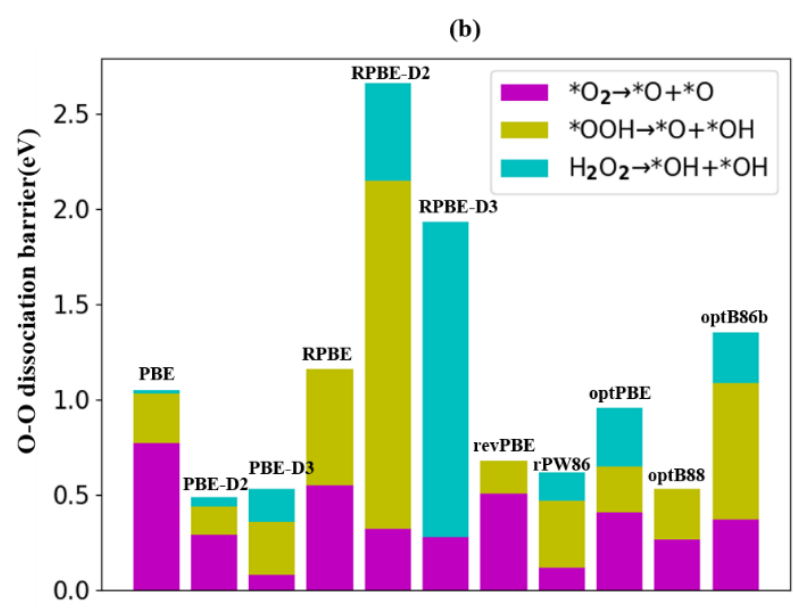

(d)

Figure 9: (a) ORR limiting potential plotted against *OH adsorption free energy with reference to the experiment ${ }^{79}$, (b) rate of limiting step in logarithmic scale along the most facile ORR pathway, (c) selectivity between four-electron vs. two-electron ORR and (d) activation energy barriers for chemical O-O bond dissociation steps for ${ }^{*} \mathrm{O}_{2}$, $* \mathrm{OOH}$, and ${ }^{*} \mathrm{H}_{2} \mathrm{O}_{2}$ intermediates for all the DFT methods considered.

The highest agreement with the experimental ORR limiting potential is found for PBE, PBE-D2, PBE-D3, and optPBE methods. The highest limiting potential predicted by RPBE-D2 method is arising from the reverse binding trend predicted for $* \mathrm{O}_{2}$ and $* \mathrm{OOH}$ binding. It is important to notice that the thermodynamic limiting step associated with these methods show a considerable variance $\left(\mathrm{PBE}: \mathrm{O} \_\mathrm{S} 1+* \mathrm{OH} \_\mathrm{S} 2 \rightarrow * \mathrm{OH} \_\mathrm{S} 1+* \mathrm{OH} \_\mathrm{S} 2 ; \mathrm{PBE}-\mathrm{D} 2, \mathrm{D} 3: * \mathrm{OH} \_\mathrm{S} 1 \rightarrow \mathrm{H}_{2} \mathrm{O}\right.$; optPBE: $\left.* \mathrm{O}_{2} \_\mathrm{S} 1 \rightarrow * * \mathrm{OOH} \_\mathrm{S} 1\right)$ but regulated by a similar $* \mathrm{OH}$ binding strength either directly or via 
scaling relations observed as evident from the linearity of the $\Delta \mathrm{GOH}$ vs. $\mathrm{U}_{\mathrm{L}}$ relation. It is surprising that the optimum $* \mathrm{OH}$ binding corresponding to the experimental limiting potential is very close to that observed from the $\Delta \mathrm{GOH}$ vs. $\mathrm{U}_{\mathrm{L}}$ relation reported by Kulkarni et al. for a set of metal catalysts. ${ }^{80}$ This can be rationalized based on the similarity in the rate-limiting $* \mathrm{OH}_{-} \mathrm{S} 1 \rightarrow *+\mathrm{H}_{2} \mathrm{O}$ step as well as similar $* \mathrm{OH}$ adsorption free energy originating from scaling relations for $\mathrm{Ir}, \mathrm{Pd}, \mathrm{Pt}$ (111) surfaces in their work and similar extent of solvent assisted stabilization across these dispersion correction methods in our study (Table 1).

Despite the adequacy of a thermodynamic analysis in explaining the activity trends, it often provides an upper bound activity estimation. In this regard, an electrochemical kinetic model is designed for studying the kinetics of ORR, the details of which are provided in the supporting information (Text S1). We have computed the energy barriers for O-O bond dissociation steps for $\mathrm{O}_{2},{ }^{*} \mathrm{OOH}$, and $\mathrm{H}_{2} \mathrm{O}_{2}$ species via CI-NEB calculations, whereas an activation barrier of $0.25 \mathrm{eV}$ has been adapted for electrochemical reactions in accordance with the report by Tripkovic et al. ${ }^{81}$ The kinetic limiting steps along the most energetically facile pathway for all the methods are identified (Table S3), and the corresponding rate constants at the ORR working potential of $0.9 \mathrm{~V}$ is given in Figure 9b. It can be seen that the activity trend predicted from kinetics is in agreement with thermodynamically predicted trends which corroborates the observation by Hansen et al. for Pt(111) surface. PBE, PBE-D2, RPBE-D2, and optPBE methods are occupying the higher most kinetic activiy region as observed in the thermodynamic investigation. However, it is to be noted that for PBE and rPW86 methods, there is a shift in thermodynamic and kinetic limiting steps from $* \mathrm{O}$ hydrogenation to $* \mathrm{O}_{2}$ hydrogenation and from $* \mathrm{OH}$ hydrogenation to $* \mathrm{O}_{2}$ hydrogenation, respectively. This suggests that potential dependent activation barrier investigation could be important in correctly identifying the steps determining the ORR kinetics. The selectivity between $4 \mathrm{e}^{-}$and $2 \mathrm{e}^{-}$oxygen reduction is analyzed by comparing the rates of $* \mathrm{OOH} \rightarrow * \mathrm{O}+\mathrm{H}_{2} \mathrm{O}$ and $* \mathrm{OOH}$ $\rightarrow \mathrm{H}_{2} \mathrm{O}_{2}$ steps as they govern the further pathways followed by **OOH (Figure 9c). Except for RPBE-D3, revPBE and rPW86, all other methods are found to possess a higher selectivity towards $\mathrm{H}_{2} \mathrm{O}$ formation compared to $\mathrm{H}_{2} \mathrm{O}_{2}$. It is to be noted that we have adapted a constant electrochemical barrier and hence therefore, not compared the predicted kinetics with the experiment as obtaining transition states of all elementary steps across all the methods considered is computationally expensive. Furthermore, it is reported that the entropic effect associated with proton mobility could 
result in different experimental frequency factors and hence kinetics. ${ }^{81}$ However, since thermochemical $\mathrm{O}-\mathrm{O}$ bond dissociation steps can play a vital role in deciding the formation energetics of intermediates channeling ORR pathways, we have calculated the activation barriers associated with these steps across all the methods (Figure 9d). Our calculations revealed the least $\mathrm{O}_{2}$ dissociation barrier $(0.08 \mathrm{eV})$ for the PBE-D3 method, which is $0.69 \mathrm{eV}$ lesser than the PBE method, which implies the inclusion of dispersion interactions greatly affects the kinetics of ORR. A similar stabilization of transition state is also observed for RPBE functional where both D2 and D3 methods improve the activation energy barrier. The lower barrier observed for PBE-D3 compared to $\mathrm{PBE}$ can be attributed to the shorter bond length of $* \mathrm{O}-* \mathrm{OH}$ transition state species with the surrounding water BL, which provides higher stability and hence lowering the activation energy. The transition state configuration for PBE-D3 is provided as a representative model for other methods in Figure S9. The least limiting potential identified for RPBE-D2 associated with the limiting step ${ }^{*} \mathrm{O}_{2} \rightarrow * \mathrm{OOH}$ is well reflected in the highest barrier for ${ }^{*} \mathrm{OOH}$ dissociation. The negligible $\mathrm{H}_{2} \mathrm{O}_{2}$ dissociation barrier observed for many methods can be attributed to the formation of a more stable $* \mathrm{OH}-* \mathrm{OH}$ species on the surface, stabilizing the BL configuration. This further supports the higher selectivity observed towards $\mathrm{H}_{2} \mathrm{O}$ production as $\mathrm{H}_{2} \mathrm{O}_{2}$ formed over the surface undergoes rapid dissociation to $* \mathrm{OH}$, which would further undergo protonation to increase the total $\mathrm{H}_{2} \mathrm{O}$ production over $\mathrm{Pt}(111)$ surface. When all the three O-O bond dissociation steps are taken into account, it can be seen that within the semi-empirical framework, PBE-D2, PBE-D3, and within the vdW-DF framework, revPBE, rPW86, optPBE, optB88 methods exhibit the highest kinetic favorability. A reasonable agreement for the energetics of these steps predicted from both thermodynamic and kinetic analysis is identified for the majority of DFT methods, given the structural deviation of transition state geometries does not differ significantly from the reactant geometries. Comparing to a completely thermodynamic analysis, it can be seen that there is a larger dispersion in the $\mathrm{O}-\mathrm{O}$ bond dissociation energetics predicted by different methods. However, since the potential determining step associated with all the methods belong to the *OOH electrochemical reduction pathway, the effect of large deviations in $\mathrm{O}-\mathrm{O}$ bond dissociation kinetics turns out to be minimal. In this respect, we envision a completely thermodynamics-based approach would suffice for an accurate ORR activity prediction irrespective of the DFT method under consideration. By scrutinizing the whole set of methods considered, under both thermodynamic and kinetic considerations, PBE, PBE-D2, PBE-D3, RPBE, RPBE-D2, and optPBE methods turn 
out to be the optimal methods for ORR activity prediction. Out of these, by considering the better structural description of catalyst surface and solvent interaction, reproducibility of experimentally observed limiting potential, selectivity between $\mathrm{H}_{2} \mathrm{O}$ and $\mathrm{H}_{2} \mathrm{O}_{2}$, and reasonable $\mathrm{O}-\mathrm{O}$ bond dissociation barrier, PBE-D3 and optPBE methods emerge as the ideal dispersion corrected DFT methods for ORR activity simulation. This observation suggests that a dispersion consideration within the computationally inexpensive semi-empirical framework suffice for an accurate ORR activity simulation compared to theoretically more robust advanced van der Waal's methods.

\section{Conclusions}

We have carried out a systematic analysis investigating the role of dispersion effects for the DFT simulation of electrocatalysis by conducting a case study of the oxygen reduction reaction. Nine DFT methods belonging to semi-empirical and non-local van der Waals functional theoretical frameworks were selected, in addition to two reference PBE and RPBE functionals without any dispersion correction. Variations in the predicted structural properties such as lattice parameter, work function, and surface energy of $\operatorname{Pt}(111)$ surface have been identified with different methods where the highest correlation with experiment is observed for DFT-D3 and optimized van der Waals functionals. A hybrid solvation model cumulatively accounting for both implicit and explicit solvent effects is considered for analyzing the dispersion effects on ORR energetics. The strength of $\mathrm{H}_{2} \mathrm{O}$ monomer binding is significantly improved when dispersion interactions are included. This is further extended when water bilayer is adapted for electrolyte simulation where H-down configuration has been found to be most stable for all methods except rPW86. The structural configurations of water bilayers have been found to be significantly different with methods where RPBE-D3 and rPW86 resulted in perfect buckled bilayers. In spite of the different binding strengths observed, all the DFT methods predicted dominance of metal- $\mathrm{H}_{2} \mathrm{O}$ interaction over intra- $\mathrm{H}_{2} \mathrm{O}$ hydrogen bonding resonating with the experimental wettability identified for $\operatorname{Pt}(111)$ surface. The simulated adsorption energetics revealed higher destabilization of $* \mathrm{O}_{2}$ and $* \mathrm{O}$ species and stabilization of $* \mathrm{OH}$ and $* \mathrm{OOH}$ species owing to the disparity in the number of hydrogen bonds made between the adsorbed species and water molecules. Method-dependent scaling relation between the adsorption free energies of ORR intermediates is found to be weakened when solvent effects are applied compared to gas phase scenario due to the deviations in stabilization effects offered by different methods. However, a reasonable scaling between $* \mathrm{OH}$ 
and $* \mathrm{OOH}$ intermediates is retained across the methods. Surface stability analysis via constructing Pourbaix diagrams conveyed a higher *O coverage under ORR relevant potentials and $\mathrm{pH}$ which for all the methods. However, the relative potential windows for different monolayers of coverage of $* \mathrm{O}$ and $* \mathrm{OH}$ are found to be affected by the methods. A reasonable congruence between kinetic and thermodynamic ORR activity predictions has been observed for all the methods with *OOH reduction emerges as the most dominant pathway. PBE-D3 and optPBE methods are identified as optimum choices for the accurate prediction of ORR activity on $\operatorname{Pt}(111)$ surface from the thermodynamic and kinetic analysis, followed by PBE-D2 and RPBE-D2 methods. Our work necessitates the importance of dispersion interactions to be taken into consideration for DFT-based electrocatalytic simulation for accurate prediction of structural and energetic features. Also, it is verified that dispersion treatment at a semi-empirical method can be equivalent or even better compared to advanced van der Waal's functionals for an accurate simulation of ORR activity. These results can be expected to be valid for other electrocatalytic reactions.

\section{Associated Content}

\section{Supporting Information}

Relative stabilities between H-up and H-down water bilayer configurations, adsorption configurations of water bilayer, change in work function variation of $\operatorname{Pt}(111)$ surface and mean absolute bond length deviation of water bilayers across DFT methods, diffusion barrier trend of $* \mathrm{O}$ from fcc to hcp site, $* \mathrm{OH}$ coverage configurations, coverage dependent $* \mathrm{OH}$ adsorption free energy, adsorption configurations first order Pourbaix diagrams of vdw-DF methods, second order Pourbaix diagrams of vdw-DF methods, transition state configuration, structural details of ORR intermediates in the water bilayer network on $\mathrm{Pt}(111)$ surface, limiting potentials of ORR elementary steps, rate constants of kinetic limiting steps, details of kinetic model.

\section{Conflicts of interest}

The authors declare no conflicts of interest

\section{Acknowledgements}

We thank IIT Indore for the lab and computing facilities. This work is supported by DST-SERB, (Project Number CRG/2018/001131), and SPARC (Project Number SPARC/2018-2019/P116/SL) 
New Delhi. ASN thank Ministry of Human Resources and Development, India for research fellowship.

\section{References:}

1. Stamenkovic, V. R.; Strmcnik, D.; Lopes, P. P.; Markovic, N. M. Energy and fuels from electrochemical interfaces, Nat. Mater. 2017, 16, 57-69.

2. Markovic, N. M. Electrocatalysis: Interfacing electrochemistry, Nat. Mater. 2013, 12, 101102.

3. Seh, Z. W.; Kibsgaard, J.; Dickens, C. F.; Chorkendorff, I.; Nørskov, J. K.; Jaramillo, T. F. Combining theory and experiment in electrocatalysis: Insights into materials design. Science 2017, 355, 146.

4. Debe, M. K. Electrocatalyst approaches and challenges for automotive fuel cells. Nature 2012, 486, 43-51.

5. Shao, Y.; Park, S.; Xiao, J.; Zhang, J. G.; Wang, Y.; Liu, J. Electrocatalysts for Nonaqueous Lithium-Air Batteries: Status, Challenges, and Perspective. ACS Catal. 2012, 2, 844-857.

6. Shao, M.; Chang, Q.; Dodelet, J. P. and Chenitz, R. Recent Advances in Electrocatalysts for Oxygen Reduction Reaction. Chem. Rev. 2016, 116, 3594-3657.

7. Nie, Y.; Li, L.;Wei, Z. Recent advancements in Pt and Pt-free catalysts for oxygen reduction reaction. Chem. Soc. Rev. 2015, 44, 2168-2201.

8. Sui, S.; Wang, X.; Zhou, X.; Su, Y.; Riffatc, S.; Liu, C. A comprehensive review of Pt electrocatalysts for the oxygen reduction reaction: Nanostructure, activity, mechanism and carbon support in PEM fuel cells. J. Mater. Chem. A 2017, 5, 1808-1825.

9. Kongkanand, A.; Mathias, M. F. The Priority and Challenge of High-Power Performance of Low Platinum Proton-Exchange Membrane Fuel Cells. J. Phys. Chem. Lett. 2016, 7, $1127-1137$.

10. Nørskov, J. K.; Rossmeisl, J.; Logadottir, A.; Lindqvist, L. Origin of the Overpotential for Oxygen Reduction at a Fuel-Cell Cathode. J. Phys. Chem. B 2004, 108, 17886-17892.

11. Stamenkovic, V. R.; Fowler, B.; Mun, B. S.; Wang, G.; Ross, P. N.; Lucas, C. A.; Markovic, N. M. Improved Oxygen Reduction Activity on $\mathrm{Pt}_{3} \mathrm{Ni}(111)$ via Increased Surface Site Availability. Science 2007, 315, 493-496. 
12. Kattel, S.; Wang, G. Beneficial compressive strain for oxygen reduction reaction on Pt (111) surface. J. Chem. Phys. 2014, 141, 124713-124718.

13. Zhang, L.; Iyyamperumal, R.;. Yancey, D. F; Crooks, R. M.; Henkelman, G. Design of PtShell Nanoparticles with Alloy Cores for the Oxygen Reduction Reaction. ACS Nano 2013, 7, 9168-9172.

14. Wang, C.; Li, D.; Chi, M.; Pearson, J.;. Rankin, R. B; Greeley, J.; Duan, Z.; Wang, G.; van der Vliet, D.; More, K. L.; Markovic, N. M.; Stamenkovic, V. R. Rational Development of Ternary Alloy Electrocatalysts. J. Phys. Chem. Lett. 2012, 3, 1668-1673.

15. Shin, J.; Choi, J. H.; Cha, P. R.; Kim, S. K.; Kim, I.; Lee, S. C.; Jeong, D. S. Catalytic activity for oxygen reduction reaction on platinum-based core-shell nanoparticles: allelectron density functional theory. Nanoscale 2015, 7, 15830-15839.

16. Keith, J. A.; Jerkiewicz, G.; Jacob, T.; Theoretical Investigations of the Oxygen Reduction Reaction on $\operatorname{Pt}(111)$. ChemPhysChem 2010, 11, 2779-2794.

17. Tripkovi`ca, V.; Skúlasona, E.; Siahrostamia, S.; Nørskov J. K.; Rossmeisl, J.; The oxygen reduction reaction mechanism on $\mathrm{Pt}(111)$ from density functional theory calculations. Electrochimica Acta. 2010, 55, 7975-7981.

18. Klimeš, J.; Michaelidesa, A. Perspective: Advances and challenges in treating van der Waals dispersion forces in density functional theory. J. Chem. Phys. 2012, 137, 120901.

19. Grimme, S. Semiempirical GGA-Type Density Functional Constructed with a Long-Range Dispersion Correction. J. Comput. Chem. 2006, 27, 1787-1799.

20. Grimme, S.; Antony, J.; Ehrlich, E.; Krieg, H. A consistent and accurate ab initio parametrization of density functional dispersion correction (DFT-D) for the 94 elements H-Pu. J. Chem. Phys. 2010, 132, 154104.

21. Dion, M.; Rydberg, H.; Schröder, E.; Langreth, D. C.; Lundqvist, B. L. van der Waals Density Functional for General Geometries. Phys. Rev. Lett. 2004, 92, 246401.

22. Thonhauser, T.; Cooper, V. R.; Li, S.; Puzder, A.; Hyldgaard, P.; Langreth, D. C. van der Waals density functional: Self-consistent potential and the nature of the van der Waals bond. Phys. Rev. B. 2007, 76, 125112.

23. Tkatchenko, A.; DiStasio, Jr.; R. A.; Car, R.; Scheffler, M. Accurate and Efficient Method for Many-Body van der Waals Interactions. Phys. Rev. Lett. 2012, 108, 236402. 
24. von Lilienfeld, O. A.; Tavernelli, I.; Rothlisberger, U.; Sebastiani, D. Phys. Rev. Lett. 2004, 93, 153004.

25. Murray, E. D.; Lee, K.; Langreth, D. C. Investigation of Exchange Energy Density Functional Accuracy for Interacting Molecules. J. Chem. Theo. Comput. 2009, 5, 2754.

26. Yuan, D.; Liao, H.; Hu, W. Assessment of van Der Waals Inclusive Density Functional Theory Methods for Adsorption andSelective Dehydrogenation of Formic Acid on Pt(111) Surface. Phys. Chem. Chem. Phys. 2019, 21, 21049-21056.

27. Muttaqien, F.; Hamamoto, Y.; Hamada, I.; Inagaki, K.; Shiozawa, Y.; Mukai, K.; Koitaya, T.; Yoshimoto, S.; Yoshinobu, J.; Morikawa, Y. $\mathrm{CO}_{2}$ Adsorption on the Copper Surfaces: van der Waals Density Functional and TPD Studies. J. Chem. Phys. 2017, 147, 094702.

28. Pham, T. L. M.; Vo, D.-V. N.; Nguyen, H. N. T.; Pham-Tran, N.-N. C-H versus O-H Bond Scission in Methanol Decomposition on Pt(111): Role of the Dispersion Interaction. Appl. Surf. Sci. 2019, 481,1327-1334.

29. Ye, K.-Y.; Janik, M. J. Density Functional Theory-Based Electrochemical Models for the Oxygen Reduction Reaction: Comparison of Modeling Approaches for Electric Field and Solvent Effects, J. Comput. Chem. 2011, 32, 3399.

30. Schwarz, K.; Sundararaman, R. The electrochemical interface in first-principles calculations. Surf. Sci. Rep. 2020, 75, 100492.

31. Carrasco, J.; Klimeš, J.; Michaelides, A. The role of van der Waals forces in water adsorption on metals, J. Chem. Phys. 2013, 138, 024708.

32. Hamada, I.; Lee, K.; Morikawa, Y. Interaction of water with a metal surface: Importance of van der Waals forces. Phys. Rev. B 2010, 81, 115452.

33. Hamada, I.; Meng, S. Water wetting on representative metal surfaces: improved description from van der Waals density functionals. Chem. Phys. Lett. 2012, 521, 161-166.

34. Zhang, Q.; Asthagiri, A. Solvation effects on DFT predictions of ORR activity on metal surfaces. Catalysis Today 2019, 323, 35-43.

35. Christensen, R., Hansen, H. A.; Dickens, C. F.; Nørskov, J. K. Vegge, T. Functional Independent Scaling Relation for ORR/OER Catalysts. J. Phys. Chem. C 2016, 120, 24910-24916.

36. Xiao, B. B.; Jiangb, X. B.; Jiang, Q. Density functional theory study of oxygen reduction reaction on Pt/Pd3Al(111) alloy electrocatalyst. Phys. Chem. Chem. Phys. 2016, 18, 14234. 
37. Haile, A. S.; 1 Yohannes, W.; Mekonnen, Y. S. Oxygen reduction reaction on Pt-skin Pt3V(111) fuel cell cathode: a density functional theory study. RSC Adv. 2020, 10, 2734627356.

38. Tripkovic, V. Vegge, T. Potential- and rate-determining Step for Oxygen Reduction on $\operatorname{Pt}(111)$. J. Phys. Chem. C 2017, 121, 26785-26793.

39. Liu, S.; White, M. G.; Liu, P. Mechanism of Oxygen Reduction Reaction on Pt(111) in Alkaline Solution: Importance of Chemisorbed Water on Surface. J. Phys. Chem. C 2016, $120,15288-15298$.

40. Tripkovi`ca, V.; Skúlasona, E.; Siahrostamia, S.; Nørskova, J. K.; Rossmeisl, J. The oxygen reduction reaction mechanism on $\mathrm{Pt}(111)$ from density functional theory calculations. Electrochimica Acta 2010, 55, 7975-7981.

41. Perdew, J. P.; Burke, K.; Ernzerhof, M. Generalized Gradient Approximation Made Simple. Phys. Rev. Lett. 1997, 78, 1396.

42. Hammer, B.; Hansen, L. B.; Nørskov, J. K. Improved adsorption energetics within densityfunctional theory using revised Perdew-Burke-Ernzerhof functionals. Phys. Rev. B 1999, $59,7413$.

43. Vydrova, O. A., Voorhis, T. V. Nonlocal van der Waals density functional: The simpler the better. J. Chem. Phys. 2010, 133, 244103.

44. Klimeš, J.; Bowler, D. R.; Michaelides, M. Chemical accuracy for the van der Waals density functional. J. Phys.: Condens. Matter. 2010, 22, 022201.

45. Klimeš, J.; Bowler, D. R.; Michaelides, M. Van der Waals density functionals applied to solids. Phys. Rev. B 2011, 83, 195131.

46. Aoyama, S.; Kaiwa, J.; Chantngarm, P.; Tanibayashi, S.; Saito, H.; Hasegawa, M.; Nishidate, K. Oxygen reduction reaction of $\mathrm{FeN}_{4}$ center embedded in graphene and carbon nanotube: Density functional calculations. AIP Advances 2018, 8, 115113.

47. Zhua, B.; Lu, J.; Sakaki, S. Catalysis of core-shell nanoparticle M@Pt (M=Co and Ni) for oxygen reduction reaction and its electronic structure in comparison to Pt nanoparticle, $J$. Catal. 2021, 397, 13-26.

48. Lee, K.; Murray, E. D.; Kong, L.; Lundqvist, B. I.; Langreth, D. C Higher-accuracy van der Waals density functional. Phys. Rev. B 2010, 82, 081101. 
49. Becke, A. D. Density-functional exchange-energy approximation with correct asymptotic behavior. Phys. Rev. A 1988, 38, 3098.

50. Becke, A. D. On the large-gradient behavior of the density functional exchange energy. $J$. Chem. Phys. 1986, 85, 7184.

51. Kresse, G.; Joubert, D. From ultrasoft pseudopotentials to the projector augmented-wave method. Phys. Rev. B: Condens. Matter Mater. Phys. 1999, 59, 1758.

52. Perdew, J. P.; Chevary, J. A.; Vosko, S. H.; Jackson, K. A.; Pederson, M. R.; Singh, D. J.; Fiolhais, C. Phys. Rev. B: Condens. Matter Mater. Phys. 1992, 46, 6671.

53. Implicit solvation model for density-functional study of nanocrystal surfaces and reaction pathways. J. Chem. Phys. 2014, 140, 084106.

54. Mathew, K.; Sundararaman, R.; Letchworth-Weaver, K; Arias, T. A.; Hennig, R. G. Implicit self-consistent electrolyte model in plane-wave density-functional theory. $J$. Chem. Phys. 2019, 151, 234101.

55. Henkelman, G.; Uberuaga, B.P.; Jónsson, H. A climbing image nudged elastic band method for finding saddle points and minimum energy paths. J. Chem. Phys. 2000, 113, 9901.

56. Jo, S. K.; White, J. M. Characterization of adsorption states of atomic iodine on $\mathrm{Pt}(111)$, Surf. Sci. 1992, 261, 111-117.

57. Gossenberger, F.; Roman, T.; Tonigold, K.-F.; Groß, A. Change of the work function of platinum electrodes induced by halide adsorption. Beilstein J. Nanotechnol. 2014, 5, 152161.

58. Skriver, H. L.; Rosengaard, N. M. Surface Energy and Work Function of Elemental Metals. Phys. Rev. B 1992, 46, 7157-7168.

59. Zhou, M.; Caré, S.; King, A.; Murias, D. C.; Rodts, S.; Gerber, G.; Aimedieu, P.; Bonnet, M.; Bornert, M.; Coussot, P. Wetting enhanced by water adsorption in hygroscopic plantlike materials. Phys. Rev. Res. 2019, 1, 033190.

60. Lew, W.; Crowe, M. C.; Karp, E.; Campbell, C. T. J. Phys. Chem. C Energy of Molecularly Adsorbed Water on Clean Pt(111) and Pt(111) with Coadsorbed Oxygen by Calorimetry. 2011, 115,9164. 
61. Michaelides, A.; Alavi, A.; King , D. A. Different Surface Chemistries of Water on $\mathrm{Ru}\{0001\}$ : From Monomer Adsorption to Partially Dissociated Bilayers, J. Am. Chem. Soc. 2003, 125, 2746-2755.

62. Calle-Vallejo, F.; Mart1'nezac, J. I.; Rossmeisl, J. Density functional studies of functionalized graphitic materials with late transition metals for oxygen reduction reactions. Phys. Chem. Chem. Phys. 2011, 13, 15639-15643.

63. Nørskov, J. K.; Rossmeisl, J.; Logadottir, A.; Lindqvist, L. Origin of the Overpotential for Oxygen Reduction at a Fuel-Cell Cathode. J. Phys. Chem. B. 2004, 108, 17886-17892.

64. Carrasco, J.; Hodgson, A.; Michaelides, A. A molecular perspective of water at metal interfaces. Nature Mater. 2012, 11, 667-674.

65. He, Z.-D.; Hanselman, S.; Chen, Y.-X.; Koper, M.T.M.; Calle-Vallejo, F. Importance of Solvation for the Accurate Prediction of Oxygen Reduction Activities of Pt-Based Electrocatalysts. J. Phys. Chem. Lett. 2017, 8, 10, 2243-2246.

66. Tripkovic, V.; Vegge, V. Potential- and Rate-Determining Step for Oxygen Reduction on Pt(111). J. Phys. Chem. C 2017, 121, 26785-26793.

67. Chen, J.; Fang, F.; Luo, L.; Liu, Y.; Chen, S. Electrocatalytic $\mathrm{O}_{2}$ Reduction on Pt: Multiple Roles of Oxygenated Adsorbates, Nature of Active Sites, and Origin of Overpotential, $J$. Phys. Chem. C 2017, 121, 6209-6217.

68. Naderian, M.; Groß, A. From single molecules to water networks: Dynamics of water adsorption on Pt(111). J. Chem. Phys. 2016, 145, 094703.

69. Eslamibidgoli, M. J.; Eikerling, M. H. Electrochemical Formation of Reactive Oxygen Species at Pt(111): A Density Functional Theory Study. ACS Catal. 2015, 5, 6090-6098.

70. Tonigold, K.; Groß, A. Dispersive Interactions in Water Bilayers at Metallic Surfaces: A Comparison of the PBE and RPBE Functional Including Semiempirical Dispersion Corrections, J. Comp. Chem. 2012, 33,695-701.

71. Ganyecz, A.; Kállay, M. Oxygen Reduction Reaction on N-Doped Graphene: Effect of Positions and Scaling Relations of Adsorption Energies. J. Phys. Chem. C 2021, 125, 8551-8561.

72. Wang, Y.-Y.; Chen, D.-J.; Allison, T. C.; Tong, Y. J. Effect of surface-bound sulfide on oxygen reduction reaction on Pt: Breaking the scaling relationship and mechanistic insights. J. Chem. Phys. 2019, 150, 041728. 
73. Nair, A. S.; Ahuja, R.; Pathak, B. Unravelling Single Atom Electrocatalytic Activity of Transition Metal Doped Phosphorene, Nanoscale Adv. 2020, 2, 2410-2421.

74. Hansen, H. A.; Rossmeisl, Nørskov. J. K. Surface Pourbaix diagrams and oxygen reduction activity of Pt, $\mathrm{Ag}$ and $\mathrm{Ni}(111)$ surfaces studied by DFT, Phys. Chem. Chem. Phys. 2008, $10,3722-3730$.

75. Tripkovic, V.; Cerri, I.; Bligaard, T.; Rossmeisl, J. The Influence of Particle Shape and Size on the Activity of Platinum Nanoparticles for Oxygen Reduction Reaction: A Density Functional Theory Study. Catal Lett. 2014, 144, 380-388.

76. Hansen, H. A.; Viswanathan, V.; Nørskov, J. K. Unifying Kinetic and Thermodynamic Analysis of $2 \mathrm{e}^{-}$and $4 \mathrm{e}^{-}$Reduction of Oxygen on Metal Surfaces. J. Phys. Chem. C 2014, $118,6706-6718$.

77. Greeley, J.; Stephens, I. E. L.; Bondarenko, A. S.; Johansson, T.P.; Hansen, H. A.; Jaramillo, T. F.; Rossmeisl, J.; Chorkendorff, I.; Nørskov, J. K. Alloys of Platinum and Early Transition Metals as Oxygen Reduction Electrocatalysts. Nat. Chem. 2009, 1, $552-556$.

78. Holton, O. T.; Stevenson, J. W. The Role of Platinum in Proton Exchange Membrane Fuel Cells, Platinum Metals Rev. 2013, 57, 4, 259-271.

79. Hoogers, G.; Thompsett, D. Catalysis in proton exchange membrane fuel cell technology. Cattech 2000, 3, 106-124.

80. Kulkarni, A.; Siahrostami, S.; Patel, A.; Nørskov, J. K. Understanding Catalytic Activity Trends in the Oxygen Reduction Reaction. Chem. Rev. 2018, 118, 2302-2312.

81. Kattel, S. Wang, G. Beneficial compressive strain for oxygen reduction reaction on $\mathrm{Pt}$ (111) surface. J. Chem. Phys. 2014, 141, 124713. 
Table of Content

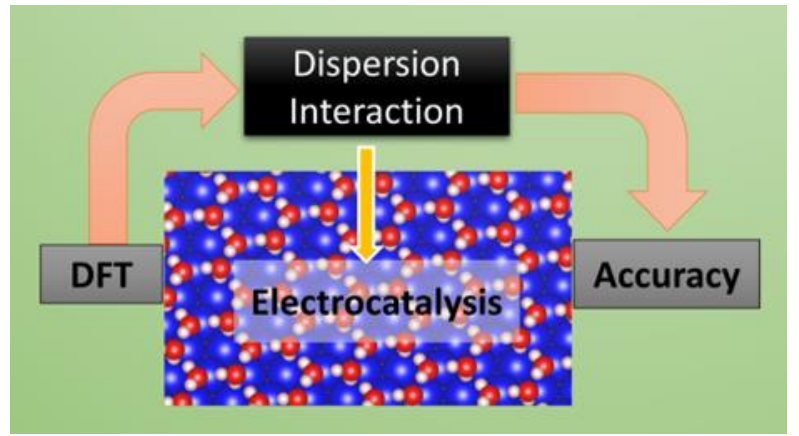

